\title{
ORDO AB CHAO. \\ COSMOGONIA E MATERIA NEL TIMEO
}

Marilisa Cannarsa*

\begin{abstract}
Secondo l'interpretazione di Migliori della modalità in cui Platone "gioca" con il lettore dei suoi dialoghi, il presente studio si confronta con alcuni fra i più "terribili giochi", nel tentativo di delineare il ruolo della "materia" nel mito cosmogonico narrato da Timeo. Attraverso un'indagine lessicale dei termini "misura", "misto" e "misurato", opereremo una distinzione tra l'attività del Demiurgo, che causa il passaggio dal caos all'ordine producendo mescolanze in giusta misura, e la passività dei misti, che esistono in virtù dell'ordine e delle proporzioni ricevute. Quanto alla distinzione tra chora ("spazio") e metaleptikôn ("materia"), la lettura approfondita del passo 51 E-52 E si focalizzerà su un uso peculiare del linguaggio riferito alla chora, specificamente alludente all'approssimazione, verosimilmente all'antica "logistica". Sulla base dei risultati dell'indagine, la progressione da una materia prima caotica ai corpi sensibili geometricamente strutturati sarà una valida chiave di lettura "dal caos all'ordine" del possibile status e della collocazione di metaleptikôn e chora in un quadro unitario del processo cosmogonico.
\end{abstract}

PAROLE CHIAVE: Cosmogonia. Misura. Misto/Mescolanza. Matéria. Spazio.

\begin{abstract}
Based on the Maurizio Migliori's interpretation of Plato's dialogues according to whom the philosopher someway "plays" with the reader of his texts - , this article intend to analyze one of the most "terrific tricks"

*Ricercatrice dell' Università degli Studi di Macerata (Italia). E-mail: mcannarsa@ unimc.it
\end{abstract}

Educ. e Filos. Uberlândia, v. 23, n. 46, p. 263-308, jul./dez. 2009. 
of his written corpus: the passage about the matter into the cosmological myth of the Timaeus. Through a lexical screening of some of the terms implicated on that "trick", we will describe the activity of the Demiourgos, who accomplish the passage from chaos to the order throug harmonic mixtures of a matter that becomes according to his craftsmanship. Besides, we'll dedicate special attention to the difference between chora ("space") and metaleptikôn ("matter") and, according to the screening's results, we'll try to demonstrate that the progression from a chaotic first matter to a geometrically structured of sensible bodies becomes a "chaos-to-order" reading key to the possible status and collocation of metaleptikôn and chora in a unit framework of the cosmogonic process.

KEYWORDS: Cosmogony. Measure. Mixture. Matter. Space.

Se dipendesse da me, fra tutti i libri antichi farei lasciare in circolazione solo gli Oracoli e il Timeo, e gli altri li farei sparire dalla vista dei miei contemporanei, perché arrivano a essere persino dannosi ad alcuni di coloro che li prendono in mano senza la dovuta preparazione.

(Proclo, presso Marino, Vita di Proclo, 38)

\section{I 'terribili giochi' del Timeo}

La celebre affermazione di Proclo in epigrafe è emblematica dell'eccezionale e durevole successo del Timeo platonico, a ragione considerato «il dialogo più letto e più studiato, e per molti aspetti anche il più influente nella storia del pensiero filosofico e teologico dell'Occidente» ${ }^{1}$. Tuttavia, osserviamo come tale successo abbia avuto un prezzo teoretico salato: l'accostamento tra Oracoli caldaici, vera e propria 'bibbia' degli antichi, e Timeo, testo di fisica ${ }^{2}$ par excellence, sancisce la

${ }^{1}$ G. Reale, Platone, Timeo, Introduzione, traduzione, note, apparati e appendice iconografica di Giovanni Reale, Rusconi, Milano 1994, Bompiani 2000, Introduzione, p. 5.

2 'Fisica' è intesa qui nel senso più ampio del termine greco physis, comprendente cioè geometria, astronomia e cosmologia, armonica, medicina e scienze naturali, e quindi 
piena 'istituzionalizzazione' dello scritto platonico, che aveva ben presto cessato di essere dialogo, divenendo trattato.

Il Timeo è, al contrario, un congegno complesso: sotto le spoglie 'enciclopediche' di summa $\pi \varepsilon p \grave{~} \varphi v ́ \sigma \varepsilon \omega \varsigma$ si celano infatti innumerevoli trappole, con frequenti rinvii e rimandi sia al suo interno, sia ad altri dialoghi del corpus Platonicum. Dal richiamo più esplicito e studiato alla Repubblica, di cui il prologo del Timeo riassumerebbe i contenuti ${ }^{3}$, alla troppo trascurata - ma a nostro parere preziosissima per comprendere Platone e l'Accademia - connessione col Filebo ${ }^{4}$, fino alla stessa bizzarra ${ }^{5}$ struttura narrativa di un dialogo tutto "scandito da ritorni all' indietro»", questo testo ci offre una testimonianza tanto inestimabile quanto a tratti inestricabile del e sul cosiddetto 'platonismo'.

Il discorso di Timeo, tradizionalmente suddiviso in tre parti, fissati gli assiomi in un vero e proprio "proemio metafisico"7, presenta una serie di passaggi difficili e tortuosi fin dalla sua prima parte incentrata sul Demiurgo o principio d'Ordine: l'inversione della trattazione di Corpo e Anima del Mondo $^{8}$, la successiva e studiatissima costituzione di quest'ultima,

abbracciante il cosmo come realtà fisica nel suo complesso.

${ }^{3}$ Sul senso di questo 'riassunto' e sulla necessità di non inquadrarlo, come fa la critica, nell'ottica di una presunta trilogia/tetralogia Repubblica-Timeo-Crizia-Ermocrate, mi permetto di rimandare al mio La 'cornice letteraria' del 'Timeo', in AA. VV., La sapienza di Timeo. Riflessioni in margine al 'Timeo' di Platone, ed. L. M. Napolitano Valditara, Vita e Pensiero, Milano 2007, pp. 3-48.

${ }^{4}$ Tale fondamentale dialogo dialettico 'tardo' è infatti a nostro avviso per molti versi, impossibili in questa sede da circostanziare, 'gemello' del Timeo.

${ }^{5}$ A tale proposito Sallis parla di «sense of the strange movement of this dialogue»; cfr. J. Sallis, Chorology: on beginning in Plato's Timaeus, Bloomington, Indianapolis, Indiana University Press, 1999, p. 3.

${ }^{6}$ Come affermò Derrida, cui tale struttura ispirò l'immagine dei «ricettacoli di ricettacoli narrativi» nel saggio Chora (Khôra, Galilée, Paris 1993); cfr. J. Derrida, Chora, in Il segreto del nome, trad. it. di F. Garritano, Jaca Book, Milano 1997, pp. 74, 83.

${ }^{7}$ Sul «preludio teoretico» e i principi metafisici da porsi alla base del discorso di Timeo si veda Reale, Timeo, Introduzione, p. 12-14.

${ }^{8}$ Timeo, nella prima parte, fa in effetti diversi 'movimenti strani': inizia a parlare del Corpo del Mondo e degli Elementi, per poi introdurre l'Anima del Mondo dichiarandola «più anziana del corpo», quindi da anteporre ad esso nel ragionamento 
il discorso del demiurgo agli dèi giovani prima della produzione della specie immortale dell'anima umana, sono solo alcuni esempi dei 'terribili giochi'9 operanti in quello che è verosimilmente l'ultimo dei dialoghi platonici pubblicati ${ }^{10}$. Ma è nella seconda parte che la lettura si complica: trattando la Causa Errante, principio di Necessità, i passaggi complessi stilisticamente prolissi e grammaticalmente ambigui, che contrassegnano indelebilmente il parlato del personaggio di Timeo, illustre main character e 'quasi-monologhista' del dialogo - sono aggravati dalla enfaticamente dichiarata difficoltà dell'oggetto trattato, l'oscuro 'terzo genere'. Su questa seconda parte e sui suoi terribili giochi concentreremo la nostra attenzione, sollevando ancora una volta quello che già dalle primissime esegesi accademiche e dalle corrispondenti e stringenti critiche aristoteliche emerse come 'il problema della materia'. Quello del principio materiale della sua natura, potenza e ruolo nella costituzione demiurgica del cosmo - è infatti un tema saldamente al centro del Timeo e del dibattito fisico e metafisico, dall'antichità ad oggi. La presente riflessione sulla materia nella cosmogonia del Timeo si avvarrà di un denso contributo di Maurizio

sul cosmo svolto fino a quel momento.

${ }^{9}$ Col termine 'gioco' ci riferiamo al procedimento 'maieutico/protrettico' applicato alla scrittura, con cui Platone invita, per non dire costringe, il lettore a fare filosofia lavorando sul testo, e per il quale si veda M. Migliori, Tra polifonia e 'puzzle'. Esempi di rilettura del 'gioco' filosofico di Platone, in AA.VV., La struttura del dialogo platonico, a cura di G. Casertano, Napoli 2000, pp. 171-212 e M. Migliori, Comment Platon ècrit-il? Example d'une écriture à caractère 'protreptique', in M. Fattal (sous la direction de), La philosophie de Platon, Paris 2005, pp. 83-118 (versione italiana: Come scrive Platone. Esempi di una scrittura a carattere 'protrettico', "Annali della Facoltà di Lettere e Filosofia dell’Università di Macerata», 37 (2004), p. 249-77).

${ }^{10}$ Com'è noto, infatti, la pubblicazione delle Leggi è postuma; cfr. Diogene Laerzio, III, 37: «Alcuni affermavano che Filippo di Opunte trascrisse le Leggi che si trovavano

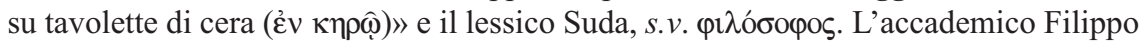
è noto principalmente come anagrapheus di Platone e come 'editore', appunto, delle Leggi; Diogene Laerzio gli attribuisce inoltre la paternità del 'tredicesimo libro', l'Epinomide (sulla questione della paternità del dialogo, cfr. lo stato degli studi in L. Tarán, Academica: Plato, Philip of Opus, and the pseudo-Platonic Epinomis, Philadelphia 1975). 
Migliori $^{11}$, che espressamente richiamiamo nel titolo del presente lavoro ${ }^{12}$ $\mathrm{e}$ in cui l'autore analizza e pone in tensione le 'ontologie del mondo sensibile'13 di Platone e Aristotele attraverso un confronto tra il Timeo e il De generatione et corruptione. Lo spunto particolare su cui intendiamo soffermarci in questa sede sono i tre schemi ontogonici che emergono dal discorso di Timeo nella trattazione dei principi di Intelligenza e Necessità. ${ }^{14}$

Com'è noto, il Demiurgo produce il cosmo fisico per mezzo di mescolanze portandolo dal disordine all'ordine ${ }^{15}$. Tale 'cosmo sensibile' o Misto prodotto è Imitazione di un modello a cui il demiurgo stesso guarda, il Paradigma o cosmo eidetico. Abbiamo così il primo schema:

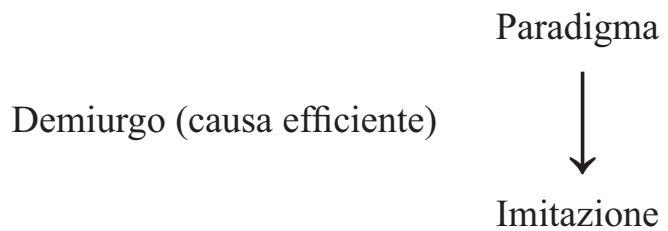

\footnotetext{
${ }^{11}$ M. Migliori, Ontologia e materia. Un confronto tra il Timeo di Platone e il De generatione et corruptione di Aristotele, in AA. VV., Gigantomachia, Convergenze e divergenze tra Platone e Aristotele, ed. M. Migliori, Morcelliana, Brescia 2002, pp. 35-104.

${ }^{12}$ Con uno 'slittamento' dall'ontologia alla cosmogonia che sarà chiaro al termine dello stesso.

${ }^{13}$ Sul concetto di 'ontologia del sensibile' (per cui si veda Giovanni Reale, Storia della filosofia antica, Vita e Pensiero, Milano 1997, vol. II, p. 452) lo stesso autore precisa: «Noi usiamo "ontologia del sensibile" proprio per ricordare il senso della fisica e della cosmologia degli antichi. Com'è noto, infatti, proprio su questo terreno si è realizzata la rottura tra pensiero moderno e concezione classica: ad una impostazione molto articolata, insieme meccanicistica e finalistica, quantitativa e qualitativa, si è sostituita la trattazione meccanicistica e quantitativa della scienza moderna. Questo fa sì che la pluralità di strumenti che Platone e Aristotele attivano, nonché tutti gli intrecci che per questa via necessariamente si determinano con gli altri ambiti, a partire da quello metafisico, sono colti con molta difficoltà dai critici»; cfr. Migliori, Ontologia e materia, pp. 35-6 e n. 2.

${ }^{14}$ I tre schemi sono stati ripresi in un ampio studio successivo; cfr. M. Migliori, La dialettica nel 'Timeo', in La sapienza di Timeo, pp. 49-107.

${ }^{15} \mathrm{Il}$ termine greco cosmos significa, appunto, 'ordine'.
} 
Lo schema, osserviamo, è di tipo 'verticale', laddove la verticalità è data dal rapporto paradigma-imitazione, mentre la causa efficiente è esterna, come nel Filebo. ${ }^{16}$

La seconda parte è espressamente un 'nuovo inizio' da 'un altro principio'. Al secondo avvio del suo mito verosimile, Timeo apre con un'oscura menzione del secondo principio e del suo rapporto col principio d'ordine, oggetto della prima parte, che non più menzionato resta sullo sfondo. Il discorso si ri-apre così sulla Necessità e sulla 'forma della causa errante, per quanto comporta la sua natura', e dunque con tutti i limiti teorici e conoscitivi di un genere disordinato, informe, privo di ragione e di misura. Migliori presenta qui due ulteriori schemi, in cui sintetizza i fattori principali o 'generi maggiori' del quadro illustrato nel primo schema 'verticale' Demiurgo-Paradigma-Imitazione, con l'aggiunta del nuovo principio della Necessità. Abbiamo così un secondo schema, di tipo 'triangolare':

\section{Dio/Causa Intelligente (sfondo) Necessità/Causa errante \\ Paradigma Ricettacolo \\ (Padre) (Madre)

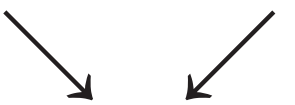 \\ Imitazione / Mescolanza}

(Figlio)

In questo schema integrato dall'aggiunta della Necessità, osserviamo un accostamento della ricettacolo-madre al paradigma-padre (i.e. le Idee come 'causa formale') che risulta alquanto spregiudicato, se pensiamo che nella prima parte l'attributo di 'padre' spetta sempre al

${ }^{16}$ Sul nô̂s come causa esterna rimando a Platone, Filebo, Introduzione, traduzione, note, apparati e appendice bibliografica di M. Migliori, Rusconi, Milano 1995, pp. 21-22 e al commentario al Filebo dello stesso autore L'uomo fra piacere, intelligenza e Bene, introduzione di Th. A. Szlezák,Vita e Pensiero, Milano 1993, Cap. XV: Metafisica e protologia, spec. pp. 448-450. 
demiurgo, qui sullo sfondo ${ }^{17}$.

Di seguito la trattazione si concentra sui diversi passaggi che portano dal caos primigenio al cosmo ordinato e sulla progressiva determinazione della 'materia prima informe'. La distinzione dei diversi stadi dell'ordinamento procede dalla causa errante alla materia informe per culminare nel cosmo ordinato/geometrizzato dall'azione demiurgica, in quello che chiameremo 'schema delle aggiunte progressive' ${ }^{18}$ :

1. Causa Errante Principio contrapposto a Intelligenza

2. Chora Spazio in cui tutto si colloca

3. Ricettacolo Materia prima informe

4. Gli elementi Determinazioni prime della materia

5. Primi corpi Determinazioni della materia e/o composizione degli

$>5$. elementi

Cosmo

Si nota immediatamente come dall'ipotesi di scansione dei processi che portano alla strutturazione dei corpi materiali emerga la vexata quaestio della natura e della potenza di chora e ricettacolo, o meglio della 'chora-ricettacolo'. Gli interpreti, infatti, mostrarono fin da subito la tendenza all'unificazione concettuale del 'terzo genere' nell'identità chora-ricettacolo, ancora oggi ${ }^{19}$ e già da Aristotele considerate «una sola e identica cosa $»^{20}$. Di contro, la novità cruciale dello schema delle aggiunte

$17 \mathrm{Su}$ questo «ennesimo tentativo di Platone di rendere più complicata la sua esposizione» cfr. Migliori, La dialettica nel 'Timeo', p. 68 e p. 97, n. 39.

${ }^{18}$ Lo schema è il frutto della lettura combinata di Timeo e Filebo, 17 A-B, 18 B-C; cfr. Migliori, Ontologia e materia, pp. 57-8.

19 Specialmente nel contesto francese: oltre all'interpretazione del già menzionato Derrida (trattata recentemente da S. Regazzoni in Nel nome di Chōra. Da Derrida a Platone e al di là, il melangolo, Genova 2008) ricordiamo fra tutte la nota definizione di Luc Brisson della chora come «milieu spatial»; cfr. L. Brisson, Le même et l'autre dans la structure ontologique du Timee de Platon: un commentaire systematique du Timee de Platon, Paris 1974. Aderisce a quest'ultima lettura nel contesto italiano Francesco Fronterotta (cfr. Platone, Timeo, Introduzione, traduzione e note di F. Fronterotta, B.U.R., Milano 2003).

${ }^{20}$ Aristotele, Fisica, IV, B, 209 b 11-16; torneremo su questo passo infra. 
progressive è che pone con forza la questione nei termini della loro distinzione, piuttosto che identificazione. Più precisamente l'emergere, nello schema costruito gradatamente sulla base della lettura analitica del testo, di molte e notevoli differenze tra ricettacolo e chora, porta l'interprete a concepire l'una (la chora) 'pre-condizione' dell'altra. ${ }^{21}$

Certamente, questa lettura ha il merito di distinguere due cose che nel testo sono effettivamente distinte ${ }^{22}$. Tuttavia, essa si scontra con una sostanziale incongruenza rispetto all'architettonica del testo stesso: l'ordine della trattazione.

Ripercorriamolo rapidamente. Il 'terzo' è introdotto da 58 righe in cui, ripetute le avvertenze sulla difficoltà del genere da trattare in aggiunta a Paradigma e Imitazione, esso è detto avere 'la potenza e la natura di un

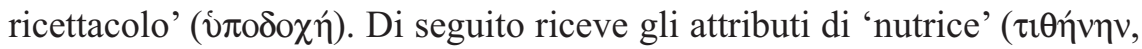

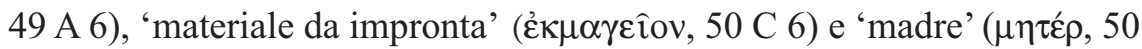

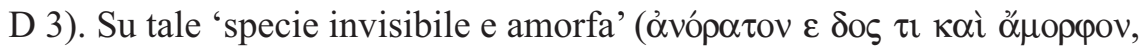
51 A 7) Timeo si dilunga fino alla riga 52 A 8 quando, 180 righe dopo il 'nuovo inizio', introduce 'a sorpresa' il terzo genere della $\chi \omega \omega_{\rho} \alpha{ }^{23}$

Appare evidente che la menzione della chora assume nel testo una collocazione che non deve passare inosservata. La sua comparsa dopo il ricettacolo 'scuote' lo schema delle aggiunte progressive: se c'è progressione, perché Timeo parla prima - e a lungo - del ricettacolo (3. nello schema) per poi improvvisamente 'aggiungere' la chora (2.)? Timeo sta forse suggerendo un parallelismo con il sopra ricordato 'gioco' dell'inversione Corpo/Anima del Mondo nella prima parte? O è così, o il quadro va rivisto.

La necessità di riformulazione dello schema delle aggiunte progressive nasce pertanto dall'incongruenza rilevata al livello dell'architettonica del testo: se l'ordine è inverso, non è al ricettacolo

${ }^{21}$ Ciò spiegherebbe l'estraneità della chora ai processi in atto nel ricettacolo, a differenza del quale essa non mostra di avere rapporti diretti con gli elementi; cfr. Migliori, Ontologia e materia, p. 71.

${ }^{22}$ Come mostreremo più dettagliatamente nel par. III.

${ }^{23}$ Timeo, anticipiamo, 'gioca' sul termine terzo: il 'terzo' è dapprima terzo rispetto alle due specie di realtà sensibile e sovrasensibile, poi è terzo insieme ad essere e generazione; v. infra, par. III. 
che spetta il 'secondo posto'? Per dare un'idea della portata teorica di un'eventuale inversione basti considerare la questione in questi termini: lo spazio è precondizione della materia, o viceversa? Esiste il luogo? Esiste il vuoto? E in questa intricata trama di problemi il linguaggio di Timeo non aiuta: la grammatica è sempre ambigua, oscura, e in virtù di ciò spesso lasciata all'arbitrio del singolo interprete ${ }^{24}$.

Per affrontare giochi così terribili con la «dovuta preparazione», non ci resta quindi che 'aggrapparci' alla struttura e all'architettonica del testo, operando come segue:

1. in primis si condurrà un'indagine lessicale dei termini 'misura', 'mescolanza' e 'misto' nell'intero testo, al fine di porre adeguatamente in evidenza la centralità e la valenza cosmogonica del rapporto Misura/misurato. Tale premessa è a nostro avviso imprescindibile per comprendere il legame: a) tra i due princìpi contrapposti di Intelligenza e Necessità; b) tra questi, il Paradigma o cosmo eidetico; c) tra questi, il Paradigma e il cosmo fisico o Misto prodotto;

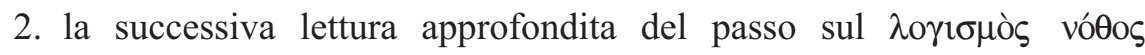
('argomento spurio' o, letteralmente, 'ragionamento bastardo') intenderà fornire una precisa ipotesi interpretativa sulla natura della chora e sul senso della sua distinzione dal ricettacolo;

3. sulla base dello screening lessicale del dialogo e del close reading

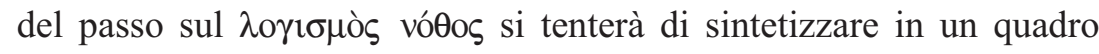
cosmogonico coerente ed unitario i tre schemi singolarmente verificati e/o riformulati.

\section{Misura, mescolanze e misti nel Timeo}

Lo screening lessicale, come abbiamo anticipato nel precedente

\footnotetext{
${ }^{24}$ Come ad esempio nella costruzione del noto passo giocato sui termini greci toûto/ toioûto ('questo/codesto'; 49 D 4-50 A 4) in cui, com'è stato osservato, «almeno due costruzioni del testo, e dunque due diverse traduzioni, della prima parte di questo celebre e difficile passo [...] sono possibili [...] grammaticalmente e sintatticamente»; cfr. Fronterotta, Timeo, p. 261-2, n. 193.
} 
paragrafo, verterà sull'uso dei termini relativi a misura, mescolanza e misto ${ }^{25}$ in tutto il Timeo, al fine di proporre, seppure in maniera inevitabilmente rapida e sommaria, una sorta di guida alla sintassi della misura nel dialogo, di cui per comodità manterremo la suddivisione 'classica' Prologo - Prima parte - Seconda parte - Terza parte, numerando per una maggiore chiarezza espositiva i passi citati.

\section{PROLOGO}

Fin dal 'prologo drammaturgico' che ospita i discorsi di Socrate e di Crizia - con un'unica, rapidissima battuta del terzo interlocutore Ermocrate - osserviamo che l'uso del termine misura assume valenza perlopiù etica, riferibile ad una corretta disposizione dell'animo umano, tanto nelle azioni (passo 1) quanto nei discorsi (passo 2):

1) 18 B 4-5: $\mu \dot{\varepsilon} \tau \rho ı \varsigma$

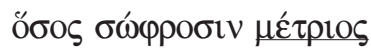

...nella misura conveniente a uomini temperati...

Contesto: Discorso di Socrate, in cui egli ricorda la necessità che i custodi della Città Ideale 'in quiete' descritta il 'giorno prima' mettano i loro beni in comune.

2) 26 A 6: $\mu \varepsilon ́ \tau \tau i \omega \varsigma$

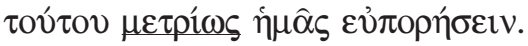

(il compito proposto ieri) saremmo stati in grado di assolverlo in modo conveniente.

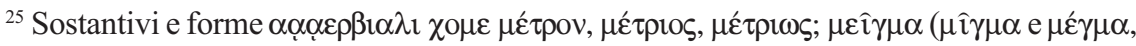

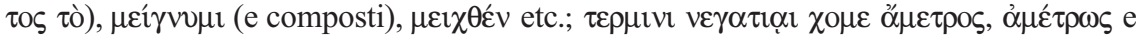

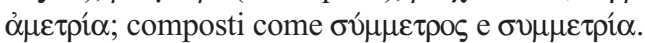

Educ. e Filos. Uberlândia, v. 23, n. 46, p. 263-308, jul./dez. 2009. 
Contesto: conclusione del Discorso di Crizia. Con queste parole Crizia accetta la proposta di Socrate di «presentare un discorso che risponda ai nostri desideri» nel banchetto di discorsi di cui si fa promotore il filosofo ateniese

\section{DISCORSO DI TIMEO: PRIMA PARTE (Principio di Intelligenza)}

Conclusi il 'prologo drammaturgico' e il 'proemio metafisico', Timeo si appresta ad esporre il suo mito/discorso verosimile. Dopo aver parlato del Corpo del Mondo e dei suoi quattro elementi (fuoco-aria-acquaterra), con una mossa repentina 'torna indietro' e descrive la mescolanza demiurgica alla base della produzione di quella che dobbiamo considerare più anziana, e non più giovane del Corpo - anteponendola pertanto in termini logico-ontologici e cosmogonici - ossia l'Anima del Mondo:

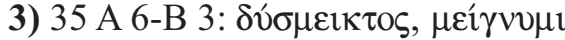

E presili tutti e tre, li mescolò tutti insieme in modo da farne una

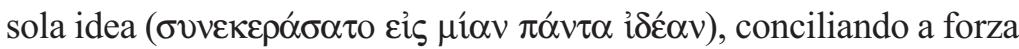
la natura del diverso che non si voleva mescolare ( $\underline{\delta} \sigma \mu \varepsilon \varepsilon \kappa \tau o v)$ a quella dell'identico. Mescolandoli poi con l'essere ( $\mu \varepsilon \imath \gamma v \grave{\varsigma} \delta \varepsilon \grave{\varepsilon} \mu \varepsilon \tau \grave{\alpha}$

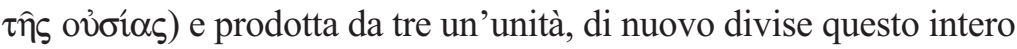
in tante parti quante conveniva, ciascuna frutto della mescolanza

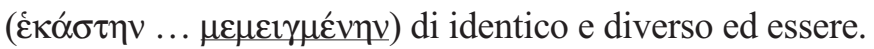

Contesto: prima, importantissima mescolanza demiurgica di

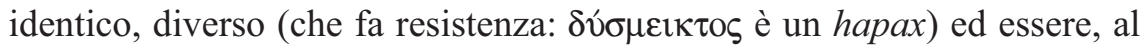
fine di produrre un'unica idea (l'anima cosmica) e da essa iniziare a dividere / recidere (i.e. 'geometrizzare'). Si osservi come la forma del verbo cambi significativamente: attiva quando l'azione è del demiurgo, medio-passiva negli altri casi (processo e prodotti). Anticipiamo che, come avremo modo di rilevare ancora, tale uso è ricorrente, regolare e a nostro parere volto ad istituire una precisa distinzione tra misura, misurante e misurato.

Poco sotto, nel medesimo contesto, troviamo infatti il participio passivo: la mescolanza è compiuta, quindi abbiamo 'il misto' (i.e. la $\mu$ í $\alpha$ 
¡ $\delta \varepsilon ́ \alpha$ come unità prodotta dalla mescolanza precedente alla divisione):

4) 36 B 5-6: $\mu \varepsilon \imath \chi \theta \varepsilon ́ v$

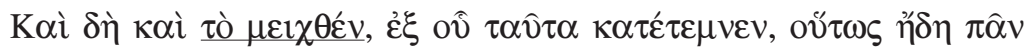
$\kappa \alpha \tau \alpha \nu \eta \lambda \omega \dot{\kappa \varepsilon l . ~}$

E il misto, da cui egli recideva, fu in tal modo adoperato tutto.

La forma attiva sembra dunque connotare in modo esclusivo e distintivo l'azione del dio, come attesta il passo immediatamente successivo:

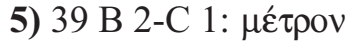

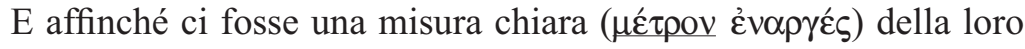
reciproca lentezza e velocità, secondo cui i pianeti procedono per le loro otto orbite, il dio accese un lume ( $\varphi \hat{\omega} \varsigma$ ó $\theta \varepsilon o ̀ \varsigma ~ \alpha ̉ v \hat{\eta} \psi \varepsilon v)$ nel secondo cerchio rispetto alla terra, che ora noi chiamiamo appunto sole, affinché illuminasse il più possibile tutto il cielo, e affinché tutti i viventi per i quali era conveniente partecipassero del numero, apprendendolo dalla rotazione dell'identico e del simile.

Contesto: creazione degli astri come strumenti del tempo. In questo passo appare la prima delle tre occorrenze in tutto il Timeo del

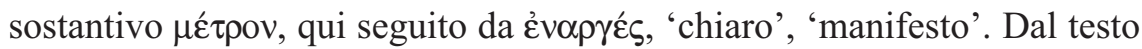
emergono chiaramente due dati: il dio accende il 'lume-sole'; le finalità di tale operazione demiurgica sono diverse e concatenate:

- stabilire una misura dei moti celesti tra loro interrelati;

- per illuminare tutto il cielo 'il più possibile';

- per permettere la partecipazione del numero a quei viventi a cui tale partecipazione sia 'conveniente' (e quindi non a tutti, il che richiama la questione, spesso sollevata da Platone nel Timeo enon solo, dell' educazione 
'appropriata', e in questo senso 'commisurata', alla natura dei discenti).

Appare anche in questo caso evidente da un lato l'attività del dio, dall'altro il limite di quanto appartiene alla sfera del divenire, che si tratti dello stesso cosmo fisico o del corpo del singolo vivente soggetto a mescolanze, com'è ben illustrato poco sotto:

6) 42 A 3-B 1: $\mu \varepsilon \mu \varepsilon \imath \gamma \mu \varepsilon \dot{v} \nu$ V

E quando le anime fossero state di necessità innestate nei corpi, e al loro corpo una cosa si aggiungesse e l'altra si separasse, per

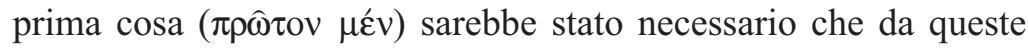
violente passioni si generasse un'unica sensazione connaturata

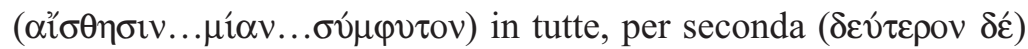
amore mescolato ( $\mu \varepsilon \mu \varepsilon \imath \gamma \mu \varepsilon ́ v o v)$ a piacere e a dolore, e oltre a questi paura e ira e tutte le altre passioni che seguono a queste, e quelle che hanno natura contraria.

Osserviamo che anche qui come nel passo 3 si passa dalla generazione di una sensazione innata, alla mescolanza delle molte passioni al livello delle parti mortali dell'anima, irascibile e concupiscibile ${ }^{26}$. Anche in questo caso la forma media del verbo rivela un uso linguistico rigoroso da parte dell'autore, cui corrisponde una netta distinzione tra soggetto 'misurante'e 'mescolante', che è sempre il dio e che sempre ha verbo attivo, e le cose misurate e mescolate, corporee, sensibili e senzienti, cui è sempre associata dall'autore la forma verbale mediopassiva. Tale uso linguisticogrammaticale rivela a nostro parere un preciso intento teoretico: il diosoggetto agisce, attuando una misura cui lui stesso guarda, e gli oggetti dell'azione demiurgica nel cosmo sensibile patiscono, in molteplici sensi e a vari livelli, a seconda del loro grado di corporeità ${ }^{27}$.

${ }^{26}$ La successione sembra inoltre richiamare la differenziazione tra sessi nelle due generazioni, la prima «uguale per tutti» e la seconda in cui nasce, appunto, «l'amore per la congiunzione»; cfr. Timeo, 91 A ss.

${ }^{27}$ Un'osservazione simile si presta indubbiamente all'obiezione di chi ritenga il richiamo alla coppia concettuale agire-patire, notoriamente aristotelico, improprio 


\section{7) 47 D 7-E 2: $\alpha_{\alpha}^{\prime} \mu \tau \tau o s$}

Contesto: in chiusura della prima parte troviamo la trattazione dei doni divini di vista e udito, in riferimento alla loro funzione e utilità per 1'uomo ${ }^{28}$. Attenzione alla successione: partito dalla vista, Timeo passa a voce e udito (46 C6-E 2): dopo la 'parola' ( $\lambda$ óyos), menziona il 'suono

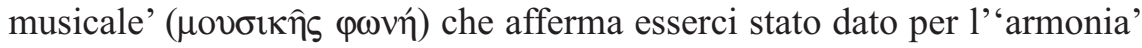

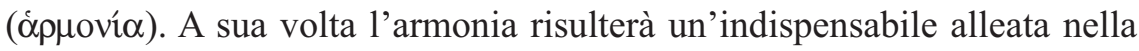
correzione dei circoli dell'anima perturbati dalla congiunzione con il

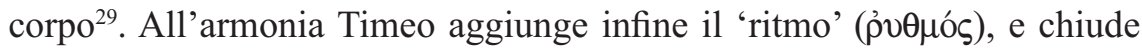
così la trattazione dell'Intelligenza:

E così, al medesimo scopo, quale aiuto per correggere quello stato

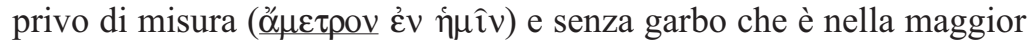
parte di noi, dalle stesse Muse ci è stato dato il ritmo.

Una prima osservazione: le Muse sono divinità mitologiche ${ }^{30}$, di livello inferiore a quello degli dèi visibili creati dal demiurgo (i.e. i corpi celesti o astri), e i loro doni all'uomo non sono così da porsi in parallelo ad essi. Non dovremmo limitarci, in altri termini, a considerare i doni divini nei

e storicamente scorretto da applicare al testo di un altro pensatore. Ad una simile obiezione risponderemmo che, se l'autore è Platone e il testo è il Timeo, ci sembrerebbe difficile ignorare gli evidenti spunti protrettici del dialogo sminuendone la portata e gli effetti nella cerchia di allievi di cui Aristotele faceva parte.

${ }^{28} \mathrm{Cfr}$. Timeo, 47 A 1-B 2: «La vista a mio giudizio è diventata per noi causa della più grande utilità, in quanto dei discorsi che ora vengono fatti intorno all'universo nessuno sarebbe mai stato fatto, se noi non avessimo visto né gli astri, né il sole, né il cielo. Ma ora, il giorno e la notte, in quanto veduto, e i mesi e i periodi ciclici e gli equinozi e i solstizi hanno realizzato (memhc£nhntai) il numero, la nozione del tempo e la ricerca intorno alla natura dell'universo. Da queste cose ci siamo procurati il genere della filosofia, del quale nessuno bene maggiore né venne né verrà mai al genere umano, essendo un dono datoci dagli dèi»

${ }^{29}$ «... avendo movimenti affini ai cicli dell'anima che sono in noi, a chi si giovi con intelligenza delle Muse non sembrerà data per un piacere irrazionale ( $\dot{\varepsilon} \varphi \hat{i}$ jovvìv

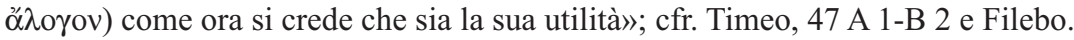

${ }^{30}$ Poco sopra, righe 40 D-41 A 3, Timeo ha parlato della generazione degli dèi della mitologia. 
termini astro (dio visibile) $:$ numero $=$ Muse (divinità mitologiche) $:$ parolasuono-ritmo, ma dovremmo stabilire una gradazione tra doni 'primari' quali la vista e l'udito, da cui derivano quelli 'secondari o derivati' della filosofia (da apprensione di numero, nozione di tempo e ricerca della natura rese possibili dalla facoltà visiva) e delle muse (percezione/produzione di suono, armonia e ritmo rese possibili dalla facoltà uditiva). ${ }^{31}$

Giunti così al termine della prima parte, riteniamo utile puntualizzare il concetto - a nostro avviso centrale - di 'utilità per l'uomo' nella sua tensione al divino: l'utile per l'uomo è qui chiaramente rappresentato dalla riconquista della misura sconvolta dall'innesto nel corpo materiale, con un'assimilazione al divino 'per quanto è possibile' ad un misto prodotto.

\section{DISCORSO DI TIMEO: SECONDA PARTE (Intelligenza/Necessità)}

La seconda parte del discorso si apre con l'esplicita aggiunta da parte di Timeo del principio della Necessità. Si osserva un uso linguistico che 'gioca' su due livelli, quello ontologico e quello del discorso, attraverso l'uso enfatico della ripetizione e il gioco di parole sull'ambivalenza, in greco, del termine $\dot{\alpha} \rho \chi \eta ́$ come principio e inizio. Timeo afferma infatti la necessità di mescolare al discorso sulla generazione del cosmo mescolanza derivante dalla persuasione dell'Intelligenza sulla Necessità - la forma della causa errante, con un nuovo inizio da un nuovo principio:

\footnotetext{
${ }^{31} \mathrm{Si}$ può certamente osservare che, in questa particolare ottica, l'apprensione del numero si situerebbe ad un livello superiore rispetto a quello dell'atto linguistico e del suono musicale: la conoscenza del numero sarebbe dunque 'più utile' di parole e suoni, e porrebbe la vista al di sopra dell'udito. Ciò non stupisce nel contesto culturale greco, in cui il senso della forma e della plasticità armonica assurge indubbiamente a cifra distintiva rispetto a culture del suono e della parola come quella ebraica. Ma anche in questo caso Platone non ci risparmia un ennesimo, stupefacente rovesciamento: se infatti questa gradazione è riscontrabile nella parte della trattazione inerente la sfera dell'intelligenza, assisteremo, nella chiusa parallela della seconda parte incentrata sulla combinazione tra intelligenza e necessità, ad un ribaltamento in cui ai colori spetta l'ultima menzione tra le impressioni sensibili, subito dopo quella dei suoni.
} 
8) 47 E 3-A 6: $\mu \varepsilon \mu \varepsilon \imath \gamma \mu \varepsilon ́ v \eta, \mu \varepsilon \imath \kappa \tau \varepsilon ́ o v$

Le cose che abbiamo detto in precedenza, tranne poche, hanno chiarito le opere prodotte dall'intelligenza; ora bisogna aggiungere al discorso anche ciò che avviene per mezzo della necessità. La generazione di questo cosmo si è prodotta infatti come mescolanza dalla combinazione di necessità ed intelligenza ( $\mu \varepsilon \mu \varepsilon i \gamma \mu \varepsilon ́ v \eta \eta$

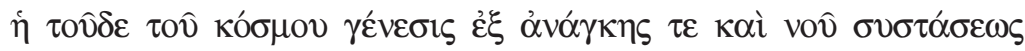
$\varepsilon ่ \gamma \varepsilon v v \eta \dot{\theta} \theta$ ) e poiché l'intelligenza dominava la necessità con il persuaderla a condurre verso l'ottimo la maggior parte delle cose che si generavano, in questo modo e per tali ragioni, per mezzo della necessità vinta dalla persuasione intelligente, da principio fu costituito questo universo. Pertanto, se si vuole dire effettivamente come il cosmo si è generato, bisogna mescolare anche la forma della

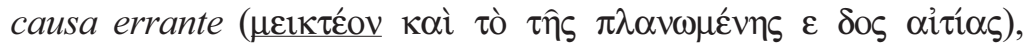
per quanto comporta la sua natura. Bisogna, dunque, che torniamo indietro, e ricominciamo di nuovo da un altro principio che si addice a queste cose. Come abbiamo fatto per le cose di allora, così anche per queste bisogna ricominciare di nuovo da principio.

9) 53 A 7-8: $\alpha \mu \varepsilon ́ \tau \rho \omega \varsigma$

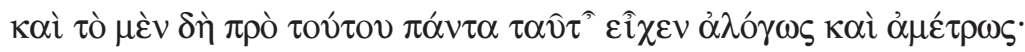

E prima di questo tutte le cose si trovavano senza ragione e senza misura.

L'affermazione si riferisce in generale alla condizione precedente l'ordinamento demiurgico; nel testo, essa segue immediatamente alla prima menzione del terzo genere come " $\chi \omega \dot{\rho} \alpha$ (sempre)». Lasciando per il momento da parte il passo, di cui tenteremo una lettura più approfondita nel successivo paragrafo, si soffermiamo sull'analogia con la 'pulitura del frumento' (53 A) per descrivere una forma di 'ordine tramite scuotimento' che le diverse parti del ricettacolo aggregarsi/allontanarsi secondo somiglianza/diversità, occupando così ciascuno un luogo ( $\chi \omega ́ \omega \alpha, 53 \mathrm{~A} \mathrm{6}$ ) 
proprio, medesimo se simili, diverso se dissimili. L'accenno è a quelli che Migliori chiama 'protoelementi', ${ }^{32}$ e che sono aggregazioni occasionali di fuoco, aria, acqua e terra - che avevano solo «qualche traccia di sé» - in uno stato comunque pre-cosmico. ${ }^{33} \mathrm{Dal}$ testo emerge così un passaggio da 'protoelementi' a 'elementi' che avviene in virtù dell'effetto della persuasione del demiurgo sul corretto scuotimento ricettacolo. Il demiurgo dunque, seppure non nominato, è tuttavia assai presente in questa seconda parte (come, dopo il suo "abbandono", le divinità inferiori) e lo è nel suo portare ordine in una realtà disordinata persuadendola attraverso la/e misura/e. Il ricettacolo è invece lo strumento scuotitore che, accogliendo in sé tutte le forme, permette l'aggregazione dei quattro elementi tramite un corretto scuotimento che porta ogni osa nel "luogo" suo proprio. La giusta misura dello scuotimento del ricettacolo va sottolineata sia in connessione con il riferimento alla persuasione dell'Intelligenza sulla Necessità, sia in vista dei prossimi e soprattutto di quanto verrò detto nella terza trattazione.

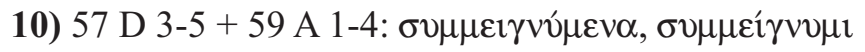

Contesto: composizione degli elementi fisici da parte del demiurgo mediante proporzioni. Timeo prima afferma che sulla questione

si deve dire che il dio, nella misura in cui la natura della necessità, per attività spontanea o ricevendo persuasione, si arrese, le abbia armonizzate, dopo averle portate a compimento in ogni parte con esattezza secondo rapporti numerici (56 C 3-7)

quindi indica come causa della molteplicità delle specie degli

\footnotetext{
${ }^{32} \mathrm{Ma}$ forse dovremmo più correttamente denominarli 'protocorpi', in quanto Timeo dirà che i veri 'elementi' sono i triangoli isoscele e scaleno, mentre qui si sta parlando dei quattro elementi 'classici' della tradizione cosmogonica dei presocratici.

${ }^{33}$ Cfr. Timeo, 53 B 1-5: «quando il dio intraprese a ordinare l'universo, il fuoco in primo luogo e la terra e l'aria e l'acqua, avevano sì qualche traccia di sé, ma si trovavano in quella condizione in cui è naturale si trovi ogni cosa quando il dio è assente; queste cose che allora si trovavano in questo stato egli in primo luogo le ordinò ( $\delta \varepsilon \sigma \chi \chi \mu \alpha \tau i$ $\sigma \alpha \tau$ ) con forme e numeri».
} 
elementi «la costituzione di ciascuno dei due triangoli elementari, perché mediante ciascuna composizione non si è prodotto da principio il triangolo di una grandezza sola, ma più grandi e più piccoli, e tanti di numero quante sono le sottospecie dei generi:

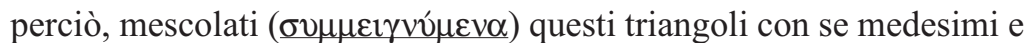
tra di loro, ne risultano infiniti per varietà.

Lo stesso composto del verbo occorre nel passo successivo, in cui si trattando le varietà delle forme di fuoco, aria e acqua, si afferma che

di nuovo, uscendo il fuoco di lì, in quanto non esce nel vuoto, l'aria vicina spinta da esso e a sua volta sospingendo la massa liquida ancora mobile verso i luoghi lasciai dal fuoco stesso, la comprime in se medesima ( $(0 \mu \mu \varepsilon \dot{\varepsilon} \gamma v v \sigma u v)$.

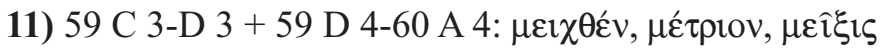

Invece, dalla terra mista $(\mu \varepsilon \imath \chi \theta \dot{\varepsilon} v)$ con esso, quando essendo invecchiata di nuovo si separa dagli altri (elementi), divenendo visibile per sé, si chiama verderame.

Sulle altre cose di questo genere, non è una cosa complicata

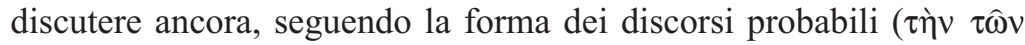

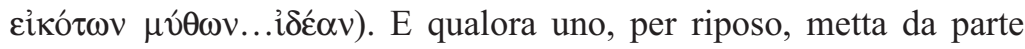
i ragionamenti su ciò che è sempre e, perseguendo ragionamenti probabili (દikó $\alpha \varsigma$ ) sul divenire, si procurasse un piacere che non gli dia poi pentimento, costui si procurerebbe un gioco moderato e

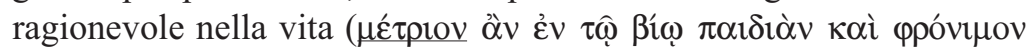
$\pi$ oเoîto). E ora, lasciandoci andare per questa via, dopo questo che s'è detto, esporremo su queste stesse cose le altre probabilità (દỉkó $\tau \alpha)$ nel modo che segue.

Contesto: si parla ancora delle molteplici varietà di forme di fuoco, aria e acqua come mescolanze che portano alle trasformazioni reciproche degli elementi, che sono tutti presenti in misura differente e determinante 
nelle varie specie. Timeo - ribadendo che su queste cose non è complicato discutere «seguendo la forma dei miti verosimili», pone l'accento sulla non-necessarietà delle conoscenze fisiche, che sono solo «probabili». ${ }^{34}$

Nelle righe immediatamente successive troviamo altre mescolanze analoghe:

L'acqua mescolata al fuoco ( che si dice fluida per il movimento e il corso che assume scorrendo sopra la terra, e si dice anche molle perché le sue basi, essendo meno stabili di quelle della terra, cedono.

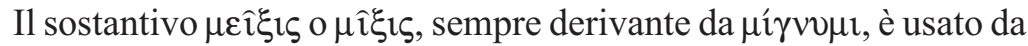
Platone 2 volte nel Timeo (e 14 nel Filebo). Leggiamo anche qui l'uso della forma media come passività degli elementi a fronte dell'azione demiurgica di mescolanza e divisione (abbiamo già detto come la forma attiva del verbo occorra sempre quando il soggetto, spesso espresso, è ò $\theta \varepsilon o ́ s)$.

Osserviamo che la ripetuta connessione tra instabilità degli elementi e (capacità di) denominazione solleva una questione interessante anche per i suoi sviluppi accademici successivi, la questione cioè dell'infinita varietà come problema da connettersi a quello della corretta denominazione di ciò che è soggetto al divenire. Se si pensa ad esempio alle specie di aria, di cui una (la più pura) è, proprio nel Timeo l'etere - osserviamo che al livello degli elementi la mescolanza è fondamentale per rendere ragione della gamma ampissima di varietà degli stessi. ${ }^{35}$

\footnotetext{
${ }^{34}$ Cfr. Timeo, 59 C 6 -D 3; questa precisazione è un esempio interessante della tecnica di scrittura platonica, che spesso inserisce queste indicazioni metodologiche determinanti in trattazioni 'tecniche'; in questo caso nella lunga 'sezione' su elementi e specie derivate, problema ontogonico ma anche gnoseologico, e qui connesso all'atto della denominazione.

${ }^{35}$ Gamma così ampia che Timeo giunge a parlare di specie addirittura non denominate: «La maggior parte delle specie di acqua che sono mescolate tra loro ( $\mu \varepsilon \mu \varepsilon i \gamma \mu \varepsilon ́ v \alpha$ $\dot{\alpha} \lambda \lambda \hat{n} \lambda$ ors) - che prese tutte insieme nel loro genere, in quanto sono filtrate attraverso le piante della terra, si dicono succhi - per le mescolanze ( $\left.\delta \grave{\alpha} \delta \tau \grave{\alpha} \varsigma \mu \varepsilon^{\prime} \hat{\imath}_{\xi} \varepsilon ı \zeta\right)$ essendo ciascuno di questi diverso dagli altri, molti costituirono specie senza nome, ma le quattro specie che contengono fuoco, essendo note più di tutte, ricevettero nomi».
} 
12) 62 C 8-D 4: $\mu \varepsilon^{\prime} \tau \rho o v$

Infatti, essendo il mondo intero di forma sferica, tutte le cose che distano ugualmente dal centro sono all'estremità, e per natura debbono essere all'estremità tutte allo stesso modo; e il centro,

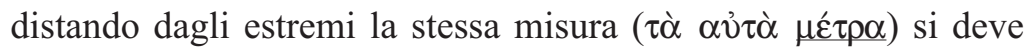
considerare come opposto a tutte.

Contesto: volendo spiegare il pesante ed il leggero, Timeo li esamina in relazione all'alto e al basso, e pone in evidenza la non-sensicità dell'attribuzione di 'alto' e 'basso' ad un cosmo sferiforme (63 A 5-6) e la necessità di comprendere da dove siano sorti questi nomi ponendolo in relazione al 'luogo' che i quattro corpi vanno ad occupare per loro natura. Il termine 'misura' assume qui un significato 'tecnico', geometrico.

13) 63 E 10-64 A 1: $\mu \varepsilon \imath \chi \theta \varepsilon \hat{\imath} \sigma \alpha$

Infatti la durezza mista $(\mu \varepsilon \imath \chi \theta \varepsilon \hat{\imath} \sigma \alpha)$ alla difformità produce il rugoso, mentre l'uniformità [mista] a densità produce il liscio.

Sempre sulla funzione produttiva delle mescolanze, cause della varietà al livello del sensibile.

14) 65 D 4-E 4: $\mu \varepsilon ́ \tau p l o \varsigma$

Contesto: i sapori come «impressioni proprie della lingua».

Timeo afferma che le impressioni chiamate «amare» sono quelle

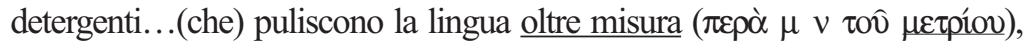

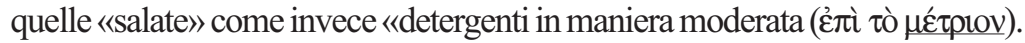

15) $67 \mathrm{C} 7: \sigma u ́ \mu \mu \varepsilon \tau \rho o s$

Contesto: i colori, quarto generi di impressioni sensibili. 
Timeo li definisce'commisurati' alla vista:

fiamma proveniente dai singoli corpi, che ha particelle commisurate

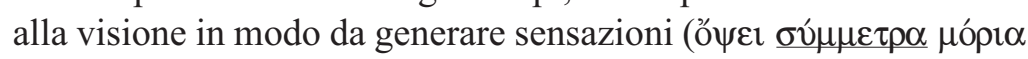

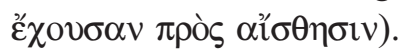

La descrizione dei colori è posta immediatamente dopo quella dei suoni, rovesciando l'ordine in cui le affermazioni sulla funzione e l'utilità della vista e dell'udito avevano chiuso la prima parte del discorso. Si può pertanto osservare che, rispetto alla prima trattazione (che era dal punto di vista della Causa Intelligente), il percorso appare rovesciato: tutto è descritto dal punto di vista della Necessità. Tale rovesciamento va a nostro parere inteso nel senso di un crescendo dal disordine all'ordine, dall' $\alpha \mu \varepsilon \tau p^{\prime} \alpha$

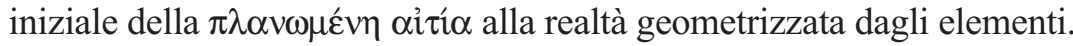

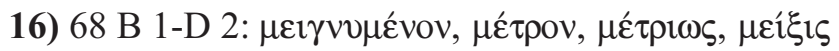

Quel genere di fuoco, poi, che è in mezzo a questi, giunge fino all'umore degli occhi e si mescola con esso, ma non è scintillante: a tale raggio del fuoco che si mescola ( $\mu \varepsilon \imath \gamma v 0 \mu \varepsilon ́ v o v)$ attraverso l'umore, e che produce un colore sanguigno, diamo il nome di rosso. Il colore splendente, poi, mescolato ( $\mu \varepsilon \imath \gamma \nu \nu \mu \varepsilon ́ v o v)$ al rosso e al bianco genera il giallo.

Ma il dire in che misura ( $\mu \varepsilon ́ \tau p o v)$ debba essere ciascuno non è cosa ragionevole neppure se lo si sapesse, perché nessuno sarebbe

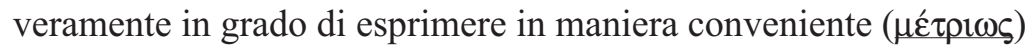
alcuna ragione necessaria e nemmeno alcuna probabile.

Il rosso, poi, mescolato al nero e al bianco, dà origine al purpureo. Si origina, invece, il colore bruno, quando si aggiunga altro nero a questi colori mescolati e bruciati. Il rosso arancione, poi, nasce dalla mescolanza di giallo e di grigio mentre il grigio nasce dalla mescolanza di bianco e di nero, e il color ocra dalla mescolanza di bianco e di giallo. Il bianco, poi, combinandosi con lo splendente e incontrandosi con il nero carico, produce il colore turchino; e il turchino, mescolandosi con il bianco, produce il celeste, mentre, 
mescolandosi al nero, il verde tenero.

Per quanto riguarda gli altri colori, da queste cose che si sono dette è abbastanza chiaro a quali mescolanze ( $\mu \varepsilon \hat{\varepsilon} \xi \varepsilon \sigma v v)$ si possono assimilare, mantenendo il mito verosimile.

Contesto: Timeo tratta qui la questione incrociata di generazione e denominazione dei colori, con un'affermazione 'strana' sulla misura e un nuovo richiamo metodologico alla verosimiglianza del mito cosmogonico. Da simili osservazioni emerge con forza il limite intrinseco del cosmo fisico, misto prodotto: tentare di dire una qualche misura della mescolanza alla base dei diversi colori non sarebbe neppure 'ragionevole', quand' anche esatta. Si osservi inoltre a che punto sia calibrata l'esposizione, scandita da richiami metodologici che chiudono e aprono le diverse trattazioni, o fungono da connettivi interni.

\section{DISCORSO DI TIMEO: TERZA PARTE $($ Misto = Uomo)}

17) 69 B 2-5: $\sigma \hat{v} \mu \mu \varepsilon \tau \rho \alpha, \sigma v \mu \mu \varepsilon \tau \rho^{\prime} \alpha$

Come s'è detto anche da principio, poiché queste cose erano disordinate il dio introdusse in ciascuna proporzioni interne e

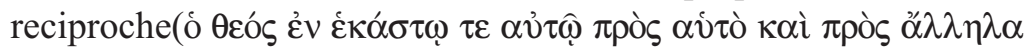

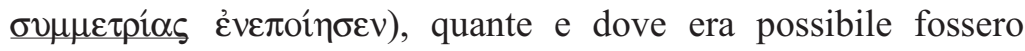

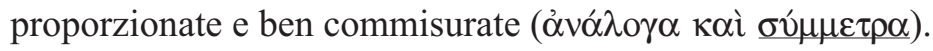

Contesto: anche nell'incipit della terza parte ci si richiama a quanto detto in precedenza, a conferma della forte unità delle tre trattazioni seppure nella loro complessa articolazione.

Si osserva anche qui il medesimo uso del sostantivo ò $\theta \varepsilon o ́ s$ con verbo attivo, ennesimo richiamo all'attività 'produttrice in quanto ordinatrice' della divinità. 


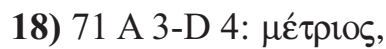

Contesto: terza parte, composizione e finalità del fegato. ${ }^{36}$

Dopo aver detto che il dio, sapendo che l'anima appetitiva/ concupiscibile «non avrebbe compreso la ragione», o che avendone eventualmente una qualche percezione non se ne sarebbe, per sua natura, curata, volendosi opporre a ciò compose «la forma (i $i \delta ́ \alpha v)$ del fegato e la collocò nella sua dimora»:

affinché la potenza dei pensieri che proviene dall'intelligenza, riflettendosi in esso come in uno specchio che riceve le forme e fa vedere le immagini, facesse paura alla forma concupiscibile dell'anima, quando servendosi dell' amarezza affine, quella potenza, penetrando grave e minacciosa, mescolandola ( $\hat{\mathfrak{i} \pi \mathrm{o} \mu \varepsilon \imath \gamma v \hat{v} \sigma \alpha)}$ rapidamente per tutto il fegato...

Al contrario, quando un'ispirazione con mitezza, provenendo dall'intelligenza, vi dipinge le immagini contrarie [...] allora tale ispirazione rende mansueta e serena la parte dell'anima che si trova presso il fegato, in maniera che durante la notte abbia una disposizione misurata ( $\left.\mu \varepsilon \tau \tau^{\prime} \alpha \mathbf{\alpha}\right)$ e durante il sonno faccia uso della divinazione, perché non partecipa di ragione e saggezza.

Dal punto di vista contenutistico-filosofico, è interessante notare che anche alla forma più bassa di anima è concesso un contatto con l'intelligenza e, conseguentemente, la ricezione da essa -per mezzo della 'paura' - della 'giusta misura'. In tale ottica, si può a nostro avviso parlare di 'persuasione intelligente che si adatta alle circostanze'. Dal punto di vista formalegrammaticale, osserviamo anche qui l' uso di i $\pi$ o $\mu \varepsilon \imath \gamma v v \hat{\sigma \alpha}$ (altro composto di $\mu \varepsilon^{\prime} \gamma v v \mu$ ) che ha come soggetto agente la «potenza dell'intelligenza»,

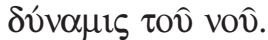

\footnotetext{
${ }^{36}$ Brano molto lungo, in cui la menzione (e la mia citazione) della misura è limitata alle ultime righe: manca un punto in un periodo di 24 righe. Al di là del tema che stiamo trattato in questa sede, sembra giusto segnalarlo come esempio del peculiare stile di questo testo.
} 


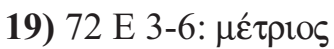

Coloro che composero la nostra stirpe ben conoscevano l'intemperanza ( $\dot{\alpha} \kappa o \lambda \alpha \sigma i \alpha \nu)$ che in noi ci sarebbe stata per le bevande e per i cibi, e che per golosità ( $\delta$ ì̀ $\mu \alpha \rho \gamma o ́ \tau \eta \tau \alpha)$ ne avremmo fatto uso molto più del giusto e del necessario ( $\mu \varepsilon \tau$ píov

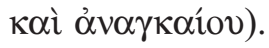

Contesto: produzione e utilità degli intestini.

La stirpe mortale, per impedire una vera e propria autodistruzione o 'estinzione per intemperanza', posero «ricettacolo di essi quello che chiamiamo basso ventre» (73, A 2-3), e vi avvolsero a spirale gli intestini per impedire all'uomo di nutrirsi continuamente e «a tutto quanto il genere umano» di diventare «a causa dell'insaziabilità ( $\delta$ iò $\left.\gamma \alpha \sigma \tau \rho \mu \mu \alpha \rho \gamma^{\prime} \alpha v\right)$ privo

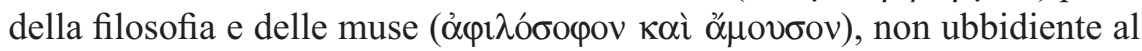
divino che è in noi» (73, A 4-8).

Siamo qui di fronte ad un ottimo esempio di persuasione dell'intelligenza sulla necessità al livello del composto psicosomatico. Il passo, piuttosto che costituire un "“atto di accusa" nei confronti della natura umana ${ }^{37}$, ci appare l'ennesimo richiamo alla 'salvaguardia della misura' a tutti i livelli, espresso nel linguaggio solenne e nel finalismo devoto ed ottimista di Timeo: 1'Artefice è ottimo, il cosmo è un dio felice, i nostri artefici sono buoni, e grazie alla loro previdenza noi non indulgiamo in eccessi alimentari autodistruttivi (di norma; ma quando lo facciamo, come dirà Timeo subito dopo, sopraggiungono naturalmente o sono indotti nel corpo $\mathrm{i}$ giusti scuotimenti del nostro ricettacolo, affinché il composto non perisca e ritorni sano). Si tratta pertanto di un ulteriore richiamo alla questione della 'giusta misura del ricettacolo', al livello del macro e del microcosmo.

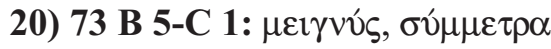

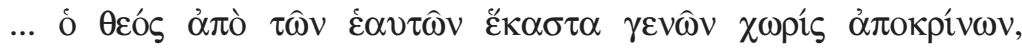

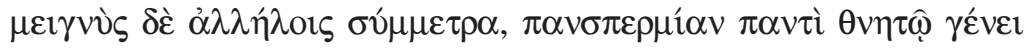
$\mu \eta \chi \alpha \nu \omega ́ \mu \varepsilon v o s, \tau o ̀ v \mu v \varepsilon \lambda o ́ v i ́$

\footnotetext{
${ }^{37}$ Fronterotta, Timeo, p. 360, n. 342. 
... il dio tenendo separati ciascuno di essi dal suo genere, li mescolò tra loro in proporzione, per produrre il seme comune a tutto il genere mortale, il midollo...

Contesto: produzione del midollo a partire dai triangoli «originari, dritti e lisci» tramite la mescolanza del dio (il verbo è attivo) che è 'in proporzione'. Il midollo come 'principio microcosmico attivo': lo sperma.

21) 74 C 5-D 2: $\mu \varepsilon \tau p i \omega s$

Contesto: i nervi e la carne. Dopo aver detto che l'utilità della carne è nel suo essere schermo per i calori eccessivi e riparo dal freddo, per difendersi «in giusta misura» ( $\left.\mu \varepsilon \tau \tau^{\prime} \omega \varsigma\right)$, Timeo afferma:

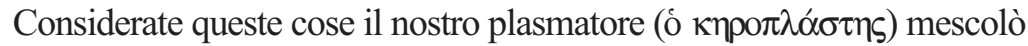

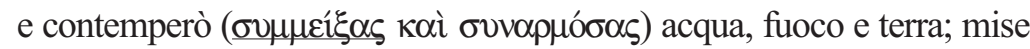
insieme un fermentato fatto di sostanze acide e salate, e mescolate ( $(\tilde{\pi} \pi \mu \mu \varepsilon \dot{\xi} \xi \alpha \varsigma)$ tutte queste cose, compose la carne succosa e molle.

Qui il soggetto del verbo (attivo) è sempre il dio, stavolta (e si

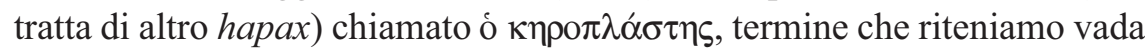

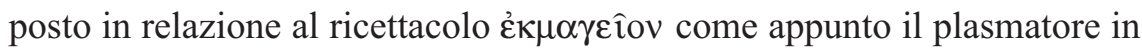
relazione alla cera; le forme verbali medie sono $\sigma 0 \mu \mu \varepsilon^{\prime} \xi \alpha \varsigma$ e $\dot{\tau} \pi \mathrm{o} \mu \varepsilon^{\prime} \xi \alpha \varsigma$, composti di $\mu$ í $\gamma \nu \nu \mu$.

22) 86 C 3-D 2: $\sigma 0 \mu \mu \varepsilon i ́ \gamma v \nu \mu 1$

Contesto: dopo aver affermato che l'anima riceve molti mali tramite il corpo a causa di umori biliosi che non trovando uscita si mescolano ( $\sigma \nu \mu \mu \varepsilon \hat{\xi} \xi \alpha \nu \tau \varepsilon \zeta, 87$ A 1) con il movimento dell'anima confondendola e causandole malattie tali da renderlo dissennato:

E colui nel cui midollo si produca un seme abbondante e impetuoso, e sia come un albero che porta frutti più del conveniente ( $\tau$ ô 


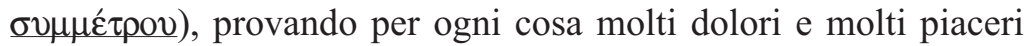

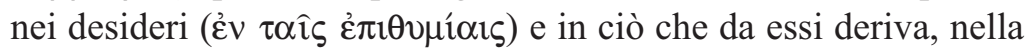
maggior parte della vita diventa esagitato ( $\dot{\varepsilon} \mu \mu \alpha v \mathfrak{s})$ proprio a causa di questi dolori e piaceri grandissimi, e pur avendo l'anima ammalata e dissennata a causa del corpo, non è ritenuto malato, ma cattivo.

Osserviamo come la malattia è descritta come un turbamento della giusta misura dovuto a piaceri smodati ${ }^{38} \mathrm{e}$ che dal corpo giunge all'anima, facendola ammalare. Timeo biasima l'errore comune del ritenere il malato nell'anima 'cattivo', e conclude con la celebre affermazione che

di sua volontà nessuno è cattivo, ma per uno stato morboso del corpo o per una crescita senza educazione il cattivo diventa cattivo

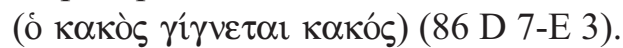

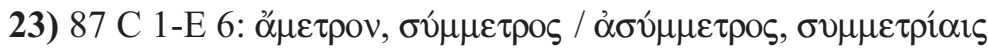

Contesto: a questo punto del testo emerge chiaramente la funzione della giusta misura al livello microcosmico del composto psico-somatico come «cura» ( $\theta \varepsilon \rho \alpha \pi \varepsilon i ́ \alpha)$ e salvezza di corpi e menti

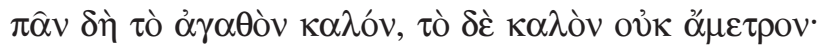

Tutto ciò che è buono è bello, e il bello non è privo di misura.

Dunque anche il vivente, per essere tale, dobbiamo supporre sia in giusta misura ( $\underline{0} \mu \mu \varepsilon \tau \rho \rho v)$.

Ora, delle giuste misure ( $\underline{\sigma} \mu \mu \varepsilon \tau p t \hat{\omega} v)$ noi avvertiamo quelle piccole $\mathrm{e}$ ce ne rendiamo conto, mentre delle più grandi e più importanti non ci rendiamo conto. Infatti, per la salute e le malattie, la virtù e

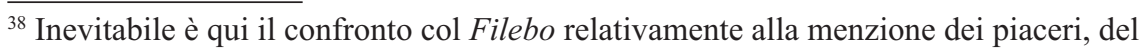
tipo di quelli che evidentemente non si accettano nella "vita mista buona". 
i vizi, nessuna giusta misura o mancanza di misura risulta essere maggiore di quella dell'anima stessa in rapporto con il corpo.

Portando l'esempio di un'anima grande e forte in un corpo piccolo e debole e viceversa, Timeo afferma che in questi casi

il vivente nel suo intero non risulta bello - privo di proporzione e

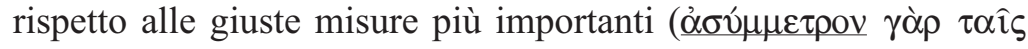

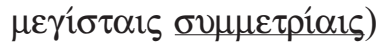

Aggiungendo l'ulteriore esempio del corpo «privo di proporzione per qualche eccesso» e quindi a) brutto a guardarsi ma anche b) causa a se stesso di mali, Timeo afferma che

dobbiamo pensare allo stesso modo dell'insieme di ambedue, che chiamiamo vivente

Si può osservare qui come, al livello del misto, l'idea di giusta misura sembri inconciliabile con qualsiasi primato si possa assegnare ad una sola delle parti del composto-uomo, in quanto il mescolato deve armonizzare e rendere simmetriche tutte le parti che compongono «il vivente nel suo intero».

24) 88 D-89 A: $\mu \varepsilon \tau p^{\prime} \omega \varsigma$

Contesto: la cura dei corpi nelle loro varie parti: necessità dell'imitazione della giusta misura della nutrice.

$\mathrm{Se}$, invece, uno imita quella che abbiamo denominato nutrice e balia dell'universo, e più che può non lascia mai che il corpo stia in riposo, nella misura del possibile, ma lo mette in movimento e imprimendo in ogni parte determinati scuotimenti, lo difende secondo natura dai movimenti esteriori ed interiori, e scuotendo in

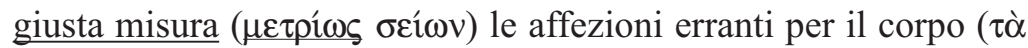




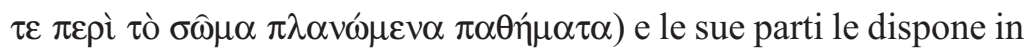
ordine ( $\varepsilon i \varsigma \tau \tau^{\alpha} \dot{\xi} \xi \mathrm{v}$ ) le une rispetto alle altre secondo affinità, in base al ragionamento che abbiamo fatto sopra intorno all'universo, non lascerà che si riproducano nel corpo guerre e malattie collocando il nemico accanto al nemico, ma farà sì che un amico posto accanto ad un amico produca salute.

Si può osservare che l'imitazione dell'uomo della giusta misura dello scuotimento del ricettacolo come $\theta \varepsilon p \alpha \pi \varepsilon i ́ \alpha$ per prevenire e curare le malattie del corpo, pone in evidenza l'utilità, per il vivente come composto psicosomatico:

- non solo di correggere i circoli dell'anima 'guastati a causa della generazione' per mezzo dei doni divini (e dunque 'provvidenziali') della vista e dell'udito, che rispettivamente permettono al vivente di apprendere ed assimilarsi - nei limiti della sua condizione - ai moti di rotazione ( $\pi \varepsilon \rho \iota \varphi \rho \rho \alpha ́)$ e all'armonia ( $\dot{\alpha} \rho \mu o v i ́ \alpha)$ celesti,

- $\quad$ ma anche di imitare la nutrice per disporre in giusta misura le 'affezioni erranti per il corpo'.

Questa duplicità nell'assimilazione non sembra adeguatamente posta in evidenza dagli approcci che tendono piuttosto a valorizzare il versante 'divino' della stessa, ignorando la prescrizione dello stesso Timeo che segnala la necessità, per la buona salute del composto, di mantenere il giusto equilibrio tra corpo e anima ${ }^{39}$.

Salvaguardia

25) 90 A 1-2: $\sigma \hat{0} \mu \mu \varepsilon \tau \rho o \varsigma$

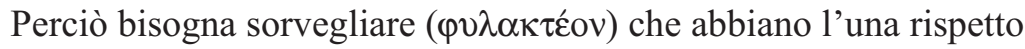
all'altra movimenti proporzionati ( $\sigma \nu \mu \mu \varepsilon \dot{\tau} \tau o v \varsigma)$.

Il passo ribadisce la necessità della salvaguardia (sorveglianza,

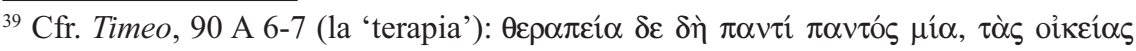

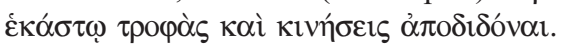


custodia) della giusta misura del composto. ${ }^{40}$ In quest'ottica, la salvezza del microcosmo umano coincide con quella del macrocosmo sensibile, e a sua volta si riconnette ad una Misura alla base della dialettica tra i due principi di ordine e disordine.

Con questo passo ci avviamo alla chiusura dello screening lessicale, da cui è emersa sia la molteplicità delle "misure", sia la loro comune derivazione da un'unica Misura portata, in quanto guardata, dalla causa intelligente, che per mezzo della persuasione riesce a penetrare il piano della necessità e a vincere così sulla causa errante, imponendo tramite un primo livello di mescolanze e divisioni una 'misura necessaria', che genera a sua volta e 'meccanicamente' molteplici "misure" e mescolanze sul piano del divenire e della realtà fisica, è cioè di quel Misto che è Figlio in quanto

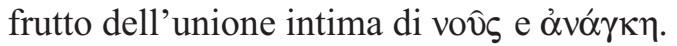

Possiamo pertanto, come fa Timeo a conclusione del suo mito/ discorso verosimile sul cosmo, chiudere la presente analisi con l'ultima occorrenza lessicale rilevata:

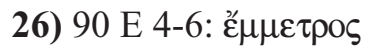

In questo modo, infatti, potremmo credere di aver mantenuto una

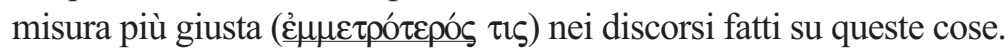

\section{Ragionare come in un sogno}

Delineato il quadro cosmogonico generale attraverso l'indagine su misura, mescolanze e misti, veniamo al problema particolare della choraricettacolo: vi è tra loro identità o sono due realtà distinte? $\mathrm{E}$ in che senso la chora - distinta o meno dal ricettacolo - è 'spazio'?

Il libro IV della Fisica di Aristotele si apre sul concetto di 'luogo' e sulle sue "molte aporie" ${ }^{41} \mathrm{Nel}$ capitolo secondo lo Stagirita fa un'affermazione ormai celeberrima: ${ }^{42}$

\footnotetext{
${ }^{40}$ Il richiamo terminologico sembra essere qui ai 'guardiani' della Repubblica.

${ }^{41}$ Aristotele, Fisica, IV, 1, 208 a 33.

${ }^{42}$ Celeberrima in quanto costituisce l'unico passo in cui Aristotele menziona quelle
} 
Perciò anche Platone afferma nel Timeo che la materia ( e lo spazio ( $\chi \omega \dot{\rho} \alpha \nu)$ sono la stessa cosa. Infatti il ricettacolo ( $\mu \varepsilon \tau \alpha \lambda \eta \pi \tau \iota \kappa o ̀ v)$ e lo spazio sono la stessa cosa. Seppure definendo il ricettacolo in modo diverso da quello delle cosiddette dottrine non scritte, ha comunque identificato il luogo ( $\tau$ ó $\pi$ ov) con lo spazio. E se tutti dicono che il luogo è un qualcosa, solo lui ha tentato di dirne l'essenza. ${ }^{43}$

E' evidente che Aristotele può rivendicare l'identità di chora e ricettacolo sulla base di una sua personale lettura del testo del Timeo. Tale 'lettura teoretica' del testo gli permette cioè di inglobarle nella sua concezione di Ǔ $\lambda \eta,{ }^{44}$ e di confutare l'assurdità in cui cadeva la controparte accademica nel porre sul medesimo piano luogo e enti matematici' ${ }^{45}$

Aldilà del serrato dibattito accademico sulla questione, quel che è certo è che per Aristotele ricettacolo e chora sono la stessa cosa. Una certa loro identificazione, come abbiamo già detto, è tuttora riscontrabile negli interpreti moderni del dialogo, seppure a diverso titolo ${ }^{46}$. Di

'cosiddette dottrine non scritte' al centro del paradigma ermeneutico della Scuola di Tubinga-Milano.

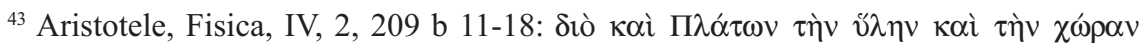

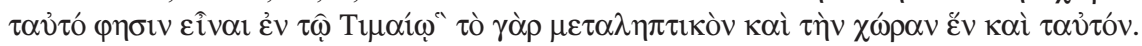

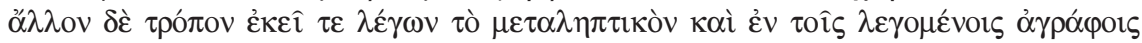

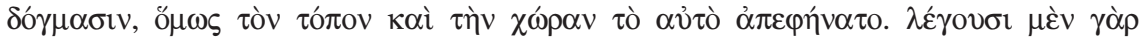

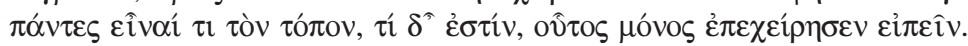

${ }^{44}$ Alcune testimonianze fanno risalire la concezione di $\tilde{} \lambda_{\eta} \eta$ a Speusippo, primo scolarca dell'Accademia e nipote di Platone. Che l'origine sia comunque accademica è indicativo della funzione 'protrettica' del Timeo (cfr. Migliori, La dialettica nel 'Timeo', p. 91-3), che porta in questo caso ad una riformulazione teorica/ridenominazione della materia.

${ }^{45}$ Cfr. Aristotele, Metafisica, XIV, N, 1092 a 17-21: «Assurdo è inoltre far nascere il luogo contemporaneamente ai solidi matematici (infatti il luogo di ciascuna singola cosa è proprio di essa, in quanto separata nel luogo, mentre gli enti matematici non hanno un luogo), e dire che essi sono in un luogo, e non dire cosa sia questo luogo». Il bersaglio dello Stagirita è l'esegesi speusippea della chora come 'luogo'; cfr. Speusippo, fr. 52 Lang, 63 Isnardi Parente ${ }^{2}, 53$ Tarán.

${ }^{46}$ Fronterotta parla di «aspetto spaziale» del «puro materiale informe»; cfr. Fronterotta, Timeo, pp. 55-62 e pp. 257-8, n. 189. Reale considera la «caratteristica della spazialità» 
contro, siamo partiti dai risultati di un recente studio da cui emergono chiaramente i termini della distinzione tra chora e ricettacolo ${ }^{47}$. Se a tale distinzione abbiamo già avuto modo di accennare nello screening lessicale complessivo del precedente paragrafo, qui si affronterà in modo più approfondito il problema della chora attraverso la lettura del passo a 51 E-52 E sul 'ragionamento bastardo' o 'argomento spurio', che è il solo a poterla afferrare.

Alla riga $51 \mathrm{D} 3$, avendo Timeo proposto di 'indagare meglio' e di optare, dovendo scegliere tra un discorso lungo e uno breve, per una «gran distinzione ben definita», Timeo si richiama alla distinzione dei generi dell'intelligenza e dell'opinione affermando:

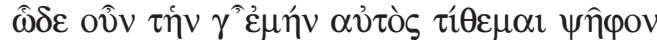

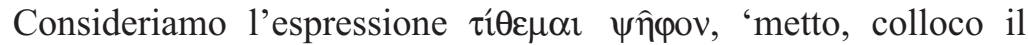
sassolino'. Il termine $\psi \hat{\eta} \varphi \circ \varsigma$ (psêfos) ha come primo significato 'pietruzza, ciottolo, sassolino'; a seconda delle operazioni possibili con esso, può significare sia 'numero, calcolo' (lat. calculus), sia 'sassolino per votare'. Le traduzioni optano per il senso traslato di "esprimere un parere"48.

Richiamati i due generi di conoscenza di intelligenza e opinione, derivanti la prima dall'insegnamento e la seconda dalla persuasione, una veritativa e di pochi, l'altra irrazionale e di tutti (51 D 3-4), si passa ai tre generi di realtà, di cui i primi due sono associati ai due generi di conoscenza, mentre del terzo si dice che guardando ad esso 'sogniamo':

un postulato o implicazione del principio materiale; cfr. Reale, Timeo, p. 24 e 151-2.

${ }^{47}$ «In sintesi, la Chòra, a differenza del Ricettacolo, non è coinvolta nei processi, appare immobile e immune, sede che non partecipa e non condivide. In questo senso, come Platone sottolinea in vario modo, essa appare davvero solo una precondizione necessaria e non sufficiente e, in un certo senso, nemmeno materiale, certamente nemmeno 'essere', collocata a fianco di essere e generazione, che a questo punto vanno intesi come segno della stabilità e della non stabilità, con essa in funzione dei processi che gli altri due termini attivano»; cfr. Migliori, La dialettica nel 'Timeo', p. 74.

${ }^{48}$ Reale traduce "ecco, dunque, qual è il mio parere"; Fronterotta scioglie la metafora con "in questa direzione, dunque, va il mio voto", riferendosi al voto dato con lo $\psi \hat{\eta} \varphi \circ \varsigma$ (in quest'ultima accezione corrisponde al ferre suffragium latino). 
51 E 6-52 B 5: Stando così le cose, si deve convenire che

(1) una è la forma identica a se stessa, ingenerata e indistruttibile, che né accoglie in sé altro da qualche altra parte, né passa mai in altro essa stessa, non visibile né altrimenti percepibile, e proprio questa l'intelligenza ebbe in sorte di guardare;

(2) e l'omonimo e omologo e a questo secondo, sensibile, generato,

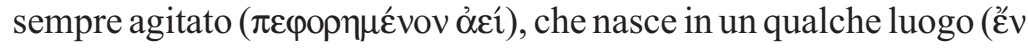
$\tau \iota v \imath \tau o ́ \pi \omega)$ e di là nuovamente perisce, comprensibile con l'opinione attraverso la sensazione ( $\delta o^{\xi} \eta ̣ n$

(3) (3) terzo ancora è il genere che è quello dello spazio sempre, che non ammette distruzione, sede di tutte le cose che hanno generazione, coglibile da un calcolo ibrido attraverso l'insensibilità

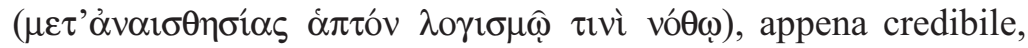
guardando al quale noi sogniamo (ỏveipo che è necessario che tutto ciò che è si trovi in un qualche luogo (๕̌v

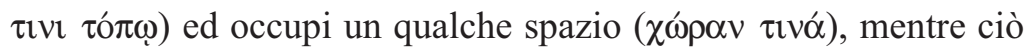
che non è in terra né da qualche parte in cielo non è affatto.

Abbiamo quindi 1) il paradigma, o cosmo eidetico, ingenerato, guardato dall'Intelligenza; 2) il cosmo fisico, generato, comprensibile con l'opinione; 3 ) il terzo genere della chora, sempre ${ }^{49}$, coglibile da un calcolo ibrido. Intelligenza, opinione e calcolo ibrido formano così una sorta di 'scala dei generi di conoscenza', ed è immediatamente evidente che, se il calcolo è ibrido, è intermedio: dunque nella 'scala' il terzo va subito spostato al posto del secondo.

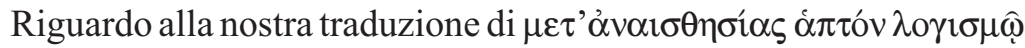

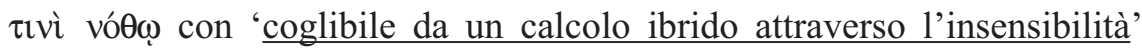
facciamo alcune osservazioni:

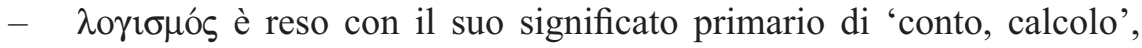

\footnotetext{
${ }^{49} \mathrm{Nel}$ greco si osserva una costruzione grammaticale piuttosto oscura ed ambigua, con ỏ ź́ giustapposto a chora, per cui è stato osservato che «Platone evita addirittura di dire che 'esiste sempre' [...], ma si limita a porre isolato $\alpha \dot{\varepsilon}$ '» in quanto lo spazio «non appartiene nemmeno alla sfera dell'essere, ma certamente a quella del 'sempre'»; cfr. Migliori, La dialettica nel 'Timeo', pp. 70-1, p. 98, n. 42.
} 
invece che con il senso secondario di 'ragionamento', innanzitutto per evitare una sovrapposizione semantica col termine greco $\lambda$ ó $\gamma$ os;

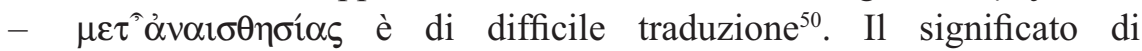

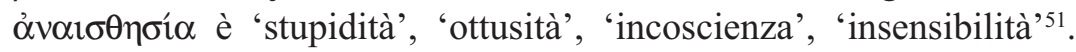
Tale 'insensibilità' è una mancanza, una deficienza, un 'non-patire'. Qui è però preceduta da $\mu \varepsilon \tau$ ó che sembra connotare l'espressione come distacco dalla sensazione, necessario e strumentale al calcolo ibrido. In altri termini, ci sembra che la privazione sia volontaria e tesa ad un fine che è il calcolo, mentre tradurre 'senza i sensi' sembra piuttosto legare tale privazione all'oscurità e all'impercettibilità dell'oggetto ${ }^{52}$.

Timeo, dunque, dopo aver parlato del terzo genere sempre come di un ricettacolo da 49 a 52, quando nomina per la prima volta la chora esprime una sorta di mònito: il terzo genere della chora è coglibile da un calcolo ibrido che si astrae deliberatamente dai sensi e che è quasi incredibile, per cui sogniamo nel momento stesso in cui affermiamo la necessità di concetti come 'luogo' e 'spazio'. Il linguaggio di Timeo è in questo passaggio enfaticamente 'onirico':

52 B 6-D 1: Tutte queste cose e le altre sorelle di queste, e intorno

\footnotetext{
${ }^{50}$ L'espressione $\mu \varepsilon \tau$ ' $\alpha \dot{v} \alpha i \sigma \theta \eta \sigma i \alpha \varsigma$ nelle traduzioni ha sempre un senso privativo/ negativo: Reale traduce «senza i sensi»; Fronterotta «che non deriva dalla sensazione».

${ }^{51}$ Platone usa il termine $\alpha \dot{v} \alpha 1 \sigma \theta \eta \sigma i \alpha \varsigma$ tre volte in tutto il corpus: due nel Timeo (52 B 3 e 74 E 8, dove si dice che la quantità di carne fu distribuita dal dio in modo

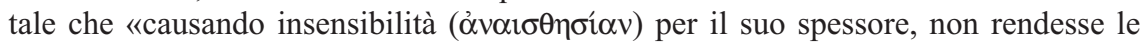
facoltà dell'intelligenza meno capaci di memoria e più ottuse») e una in Filebo, $34 \mathrm{~A} 1$, dandone qui una vera e propria definizione, come in Timeo $74 \mathrm{E}$ in un contesto riferito alla memoria e alle facoltà intellettive: «invece di dire che sfuggono all'anima, quando essa resta indifferente alle affezioni che scuotono il corpo, quello che tu ora chiami 'dimenticanza' ( $\lambda \hat{\eta} \theta \eta v)$, denominalo, invece, 'assenza di sensazione' ( $\alpha \dot{v} \alpha \imath \sigma \theta \eta \sigma i ́ \alpha v) »$; trad. Migliori.

${ }^{52}$ Optare per "con un non-qualcosa" piuttosto che per un "senza un qualcosa" significa in definitiva interpretare l'espressione come riferita al senziente che si distacca dai sensi volontariamente e fa un ragionamento 'ibrido' che è il solo che può 'afferrare' la chora.
} 
$\underline{\text { alla natura che è davvero e che non dorme, a causa di questo sogno }}$ (ỏvelp $\omega \xi \varepsilon \omega \varsigma$ ) non siamo in grado, ridestandoci, distinguendole di dire la verità, cioè che all'immagine, poiché non le appartiene neppure ciò da cui è stata generata, e sempre si aggira come fantasma di qualcos'altro, conviene perciò di generarsi in un'altra cosa, aggrappandosi in qualche modo all'essere, oppure non essere assolutamente nulla.

Timeo ci ammonisce sul pericolo di farsi affascinare dal sogno al punto da non poter dire la verità sulla 'natura che è davvero e che non dorme'. Il sogno sembra dunque una sorta di seducente allucinazione; l'immagine è un'ombra eternamente fluttuante, e Timeo aggiunge immediatamente che 'si aggrappa in qualche modo all'essere' per non essere nulla. Se il sogno non ci fa dire la verità sull'immagine, il problema che emerge sembra qui quello di una tendenza a attribuire impropriamente la statuto di realtà a qualcosa che non lo possiede. La precisazione che ci si 'aggrappa in qualche modo' rafforza questa lettura. Emerge comunque dal passo che l'immagine ha un duplice rapporto con l'essere e con la chora, in quanto la chora costituisce il sostrato necessario per cui l'immagine stessa si aggrappa all'essere ${ }^{53}$. E' a questo punto che Timeo si produce in una sorta di micidiale 'sciolilingua':

52 -D 1: Invece, a ciò che è viene in soccorso il discorso vero per la sua esattezza secondo cui finché una cosa è una cosa e un'altra è un'altra, nessuna delle due può mai divenire in nessuna delle due diventando una e insieme la stessa e due.

Il passo è molto difficile. Se 'ciò che è' corrisponde alla 'natura che è davvero e che non dorme', il discorso vero per la sua esattezza che le viene in soccorso andrà a contrapporsi al calcolo ibrido incredibile e

${ }^{53}$ «Lo status ontologico delle cose sensibili come immagini delle realtà intelligibili implica una doppia relazione ontologica: una con ciò di cui è immagine, e una con ciò in cui l'immagine si realizza. In altri termini: l'immagine implica ciò di cui è manifestazione e a cui fa riferimento come a un modello, e un sostrato o una base su cui appoggia, che è appunto la spazialità di cui stiamo parlando, e che si rende necessaria come sede di ciò che nasce (51 E-52 B)»; Reale, Timeo, Introduzione, p. 24.

Educ. e Filos. Uberlândia, v. 23, n. 46, p. 263-308, jul./dez. 2009. 
al sogno che ci impedisce di dire la verità distinguendole. Tutto il passo da 52 B 6 sembra pertanto un'avvertenza di metodo, a nostro avviso non casualmente seguita da un nuovo incipit - ma Timeo ormai ci ha abituati a questi ritorni all'indietro:

52 D 2-4: Questo, dunque, è il ragionamento che fornisce, per sommi capi, il mio pensiero. C'erano l'essere, lo spazio e la generazione, tre distinte, anche prima che si generasse il cielo ${ }^{54}$.

La chora è dunque intermedia tra essere generazione, distinta ma ugualmente precosmica.

Se la seconda asserzione non sembra dare troppi problemi di

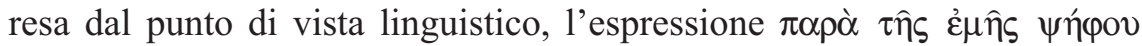

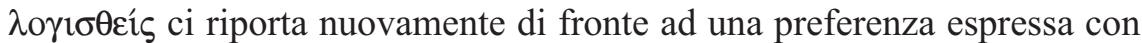
il sassolino, resa anche qui dai traduttori in senso traslato con "questo è il mio parere". Quanto al possibile significato 'matematico' del sassolino abbiamo già detto supra; il verbo che stavolta troviamo associato ad esso è $\lambda \mathrm{o} \gamma_{i} \zeta \mathrm{o \mu \alpha}$ (calcolo, conto, (e)numero, trasl. ragiono) ${ }^{55}$. E' inoltre attestata

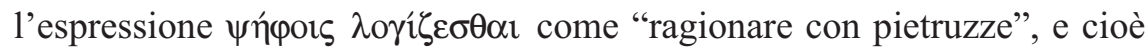
"contare", "calcolare", "numerare" 56 .

Considerando la struttura grammaticale della frase e in base a corrispondenti usi nello stesso autore ${ }^{57}$ una possibile traduzione sarebbe:

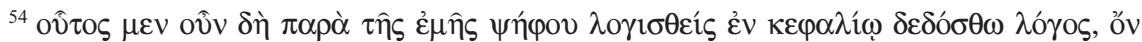

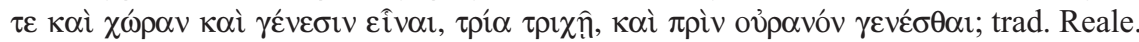
Fronterotta traduce in modo più letterale ma sostanzialmente equivalente: «questo sia dunque il discorso che riassume per sommi capi il ragionamento che ha il mio voto, che vi erano cioè l'essere, lo spazio e il divenire, tre realtà distinte, già da prima che nascesse il cielo».

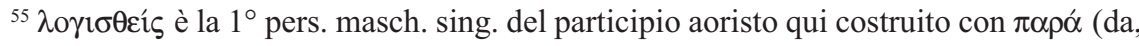

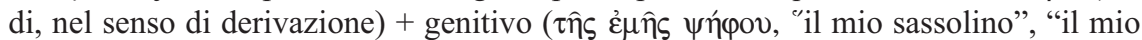
calcolo").

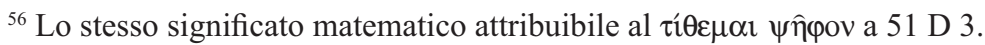

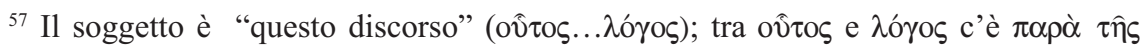

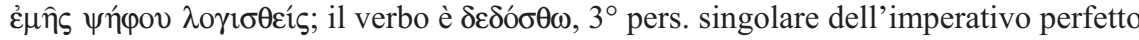
passivo di $\delta i ́ \delta \omega \mu$ ("do", "concedo") quindi: "e sia pure / sia pur concesso questo [...] discorso..." (la forma media è attestata in Platone in espressioni come "è permesso
} 
Si dia pure questo discorso per sommi capi dal mio ragionare con pietruzze, che c'erano l'essere, lo spazio e la generazione, tre distinte, anche prima che il cielo si generasse ${ }^{58}$.

Non si tratta semplicemente di optare per una sola di queste grammaticalmente possibili e apparentemente equivalenti interpretazioni del testo. Dal punto di vista storico, viene piuttosto da chiedersi se Platone non stia 'giocando' 59 con il doppio senso del termine $\psi \hat{\eta} \varphi \circ \varsigma$ e, più in generale, non stia deliberatamente adottando di un linguaggio che allude quella antica pratica di calcolo che prende il nome di logistica ${ }^{60}$. Nello specifico, a quella 'versione filosofica' della logistica che in quanto «attività teoretica facilita il passaggio dell'anima dalla sfera del divenire a quella dell'essere e della verità ${ }^{61}$. Se il linguaggio di Timeo è inteso come metafora per un ragionamento logistico ${ }^{62}$, con espressioni come 'calcolo ibrido' e

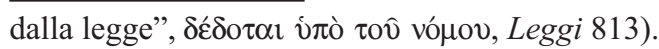

${ }^{58} \mathrm{La}$ traduzione è letterale e poco elegante, tuttavia intende rendere il grado di approssimazione a cui ci sembra alludere la formulazione di Timeo. Cfr. Migliori: «Questo, dunque, il discorso che sintetizza il ragionamento, per quel che penso».

${ }^{59}$ Intendiamo qui gioco filosofico messo in atto dell'autore che, scrivendo come scrive, da un lato apre a diverse letture possibili dello stesso testo, fungendo da irresistibile stimolo protrettico; dall'altro ammonisce in modo enfatico sulla metodologia e sui limiti del discorso. Questa tecnica di scrittura filosofica è la chiave del dibattito accademico, ieri e oggi.

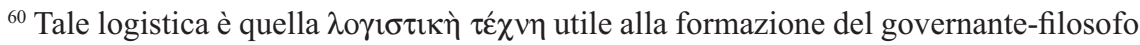
nel VII libro della Repubblica. Essa «consiste nel calcolo del numero, del pari e del dispari, come quantità in sé e nei loro rapporti reciproci» con «un'attenzione maggiore alla dimensione quantitativa, propria del calcolo, cui si contrappone la relativa maggiore formalizzazione dell'aritmetica. Ma questo non comporta una visione puramente tecnica della logistica, perché, come sappiamo, essa è duplice: la sua versione filosofica conduce l'anima ad elevarsi verso la contemplazione della pura natura del numero, abbandonando il livello dei calcoli commerciali»; cfr. M. Migliori, «Non entri chi è "ageometretos"”. Il connubio tra matematiche e filosofia nell'insegnamento di Platone, in Filosofia, logica, matematica dal periodo classico al nostro secolo. Atti del convegno dell'I.R.R.S.A.E. Marche, Ancona 25-27 marzo 1993, a cura di A. Repola Boato, Quaderni di “Innovazione Scuola”, 18 (1994), pp. 15-40, p. 22.

${ }^{61}$ Migliori, «Non entri chi è "ageometretos"», p. 22.

62 «Platone stesso sottolinea il carattere matematico delle sue considerazioni, facendo 
'ragionamento con pietruzze' si farebbe riferimento ad un esercizio mentale di tipo matematico, ossia a quella «ginnastica intellettuale orientata al bene» ${ }^{63}$ che Platone considera una disciplina intermedia tra scienza e tecnica ${ }^{64}$. In questo senso leggiamo il monito nel passo sulla 'natura che è davvero e che non dorme', di cui non si può dire il vero se non ci si risveglia dal sogno. Possiamo incidentalmente osservare che nel Timeo sembrano potersi così distinguere più 'matematiche': nella prima parte, incentrata sulla Causa Intelligente, la matematica è detta essere un dono divino all'uomo ${ }^{65}$; nella seconda parte, sulla Causa Necessaria, è logistica, un ragionamento ibrido, un calcolo approssimato e faticoso, inferiore alla dialettica fondata sul discorso vero ed esatto e che distingue, ma necessario se si vuole afferrare la chora come principio di spazialità degli elementi fisici.

Tornando al brano, vediamo che immediatamente dopo la menzione dei generi di essere, chora e generazione Timeo afferma:

52 D 4- E 1: E la nutrice della generazione, inumidita e infuocata, accogliendo in sé le forme di terra e di aria, e ricevendo tutte le altre affezioni che ad esse conseguono, appariva multiforme a

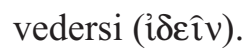

Il brano ci porta così alla domanda posta all'inizio: parlare della nutrice della generazione subito dopo la menzione della chora è un segnale

dire a Timeo, nel corso dell'esposizione, "secondo che io penso", "in breve", ma più esattamente "come mi risulta" e "tirando le somme" - cioè attribuendogli espressioni che evocano la "logistica", il disperato indaffararsi coi calcoli di cui si è già parlato. Nello stesso spirito è da intendersi la menzione dello psêfos ( $\psi \hat{\eta} \varphi \circ \varsigma)$, il ciottolo usato come contrassegno nelle operazioni aritmetiche»; R. Kaplan, Zero. Storia di una cifra, BUR, Milano 1999.

${ }^{63}$ E. Cattanei, Aristotele e i "calcoli" dell'uomo saggio, in AA. VV., Platone e Aristotele, Dialettica e logica, a cura di M. Migliori e A. Fermani, Morcelliana, Brescia 2008, pp. 61-86, p. 82.

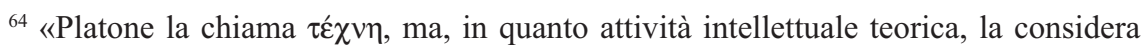

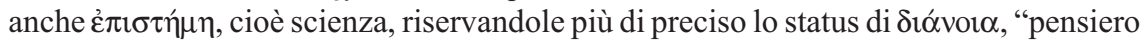
razionale discorsivo"»; cfr. Cattanei, Aristotele e $i$ "calcoli" dell'uomo saggio, p. 82 e n. 85. Cfr. il 'gioco moderato e ragionevole' visto supra, passo 11, par. II.

${ }^{65}$ La finalità della vista è nell'apprensione del numero attraverso la contemplazione degli astri, e in questo senso un dono divino; cfr. Timeo, 47 A-C.

Educ. e Filos. Uberlândia, v. 23, n. 46, p. 263-308, jul./dez. 2009. 
che chora e ricettacolo sono distinte?

Innanzitutto osserviamo che l'ỉocîv è inequivocabile: la materia è qui quella visibile e tangibile, in cui sono già presenti i corpi primi e le conseguenti affezioni, e non quella invisibile e amorfa. Da ciò sembra che la materia subisca una graduale progressione (attra)verso l'ordine, cui corrispondono fasi diverse. Migliori stesso pone in evidenza questa gradazione interna al ricettacolo, individuandone tre distinte dimensioni o livelli:

a) il Ricettacolo come materia invisibile che accoglie tutto;

b) il Ricettacolo che ha al suo interno dei protoelementi che si organizzano in qualche modo, 'per moto naturale';

c) il Ricettacolo con al suo interno le materie formate e tutte le altre cose, che entrano ed escono ${ }^{66}$.

Se consideriamo a) la materia prima, invisibile e amorfa, senza ragione e senza misura, b) il materiale da impronta in cui appaiono $i$ protoelementi e c) la materia sensibile strutturata in elementi, possiamo denominare: a) livello del Ricettacolo Intelligibile (invisibile); b) livello dei protoelementi ${ }^{67}$; c) livello del Ricettacolo Sensibile (visibile);

Il dio si serve di triangoli, forme e numeri che informano il ricettacolo, dando luogo ad aggregazioni materiali sempre più strutturate geometricamente, in una stabilizzazione formale che non può che essere 'successiva' ${ }^{68}$ allo stato caotico, inumidito e infuocato della nutrice, e che sembra avere come 'sostrato di esistenza' un qualcosa - la chora - cui è difficile riferirsi col termine di 'realtà', in quanto Timeo parla in modo oscuro: di fatto, non dice che la chora non è; tuttavia, evita di dire che è.

Possiamo allora solo ipotizzare che, se il processo di aggregazione materiale nel ricettacolo è paragonato, come fa Timeo a $52 \mathrm{D} 6$, alla pulitura del frumento, la genesi dello spazio può essere assimilabile alla spianatura del terreno fatta dal geometra per tracciarvi le sue figure. La chora sarebbe così una sorta di piano geometrico (campo o matrice) su cui il demiurgo opera per mezzo delle cause formali, e che possiamo concepire

\footnotetext{
${ }^{66}$ Migliori, La dialettica nel 'Timeo', p. 75.

${ }^{67} \mathrm{O}$ meglio, come abbiamo osservato nel secondo paragrafo, 'protocorpi'.

${ }^{68}$ La successione va intesa ovviamente in senso cosmogonico e non temporale, dato che cielo e tempo non sono ancora stati prodotti.
} 
approssimativamente come:

- implicazione strutturale ${ }^{69}$ dell'ordinamento della materia caotica tramite la giusta misura dello scuotimento al livello dei protoelementi, scuotimento che è a sua volta l'effetto della persuasione del demiurgo già avvenuta al livello del Ricettacolo Intelligibile;

- precondizione del ricettacolo che è sostrato materiale e visibile della realtà sensibile nel cosmo ordinato (livello del Ricettacolo Sensibile) ${ }^{70}$.

In questo senso essa è successiva al Ricettacolo Intelligibile, ed è pertanto da porre come terza nel 'nostro' schema delle aggiunte progressive.

Innanzitutto, in quanto spazio e luogo essa è più determinata rispetto all'indeterminatezza del caos primigenio. E' inoltre successiva perché misurata, in virtù dell'azione dei triangoli elementari, mentre nel ricettacolo solo nella dimensione intermedia i protoelementi presentano «caratteri rudimentali ${ }^{71}$ di fuoco, aria, acqua, terra.

La distinzione dei tre livelli del ricettacolo ci porta così alle seguenti conclusioni sulla chora:

- è un'implicazione dell'ordinamento 'per corretto scuotimento' del Ricettacolo Intelligibile persuaso dall'azione dell'intelligenza sulla necessità;

- è precondizione del Ricettacolo Sensibile, è sede e sostrato spaziale di tutto ciò che ha generazione sensibile;

- è coglibile da un calcolo ibrido attraverso l'insensibilità;

- guardando ad essa noi sogniamo.

Una prima modifica allo schema delle aggiunte progressive sarà allora:

${ }^{69}$ Cfr. Reale, Timeo, Introduzione, p. 24: «L'essere delle realtà intelligibili non implica affatto l'essere in qualche luogo, mentre l'essere delle cose sensibili implica strutturalmente proprio questo». Potrebbe essere pensata come il sostrato geometrico strutturale dei corpi fisici generato dal corretto aggregarsi e situarsi della materia informe persuasa.

${ }^{70}$ In questo senso leggiamo il 'ritorno al ricettacolo' di cui parla Migliori, La dialettica nel 'Timeo', pp. 71-4.

${ }^{71}$ Reale, Timeo, Introduzione, p. 24. 


1. Necessità
persuasione $\longrightarrow \quad \begin{aligned} & \text { Causa Errante come } \\ & \text { all'Intelligenza»" }{ }^{72}\end{aligned}$
giusta misura dello scuotimento antitetico
ordinamento

\section{Ricettacolo}

persuasione

3. Chora

\section{Terzo genere}

Luogo, Spazio

\section{Terzo genere}

2. a) livello del Ricettacolo Intelligibile Materia prima invisibile amorfa giusta misura dello scuotimento ordinamento

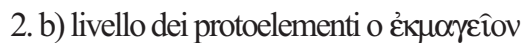

La chora dunque è il risultato di un ordinamento del caos originario: la stabilizzazione dei protoelementi genera quella spazialità «che si rende necessaria come sede di ciò che nasce» ${ }^{73}$.

Se ciò porta dunque a condividere la sottile diversità ${ }^{74}$ rilevata fra

\footnotetext{
${ }^{72} \mathrm{Si}$ osserva che a tale principio non è estranea una certa predisposizione spontanea a ricevere l'ordinamento: «caratteri rudimentali»la necessità del principio antitetico non è assoluta disteleologia, totale casualità e irrazionalità, perchè, in tal caso, non potrebbe ricevere in modo adeguato la razionalità»; Reale, Timeo, Introduzione, p. 23.

${ }^{73}$ Reale, Timeo, Introduzione, p. 24.

${ }^{74}$ «Pertanto Ricettacolo e Chòra sono diversi, certo di una diversità sottile in quanto entrambi sono precondizioni, uno con caratteristiche più materiali, l'altra più spaziali, l'uno più legato agli elementi, l'altra più genericamente condizione di tutto ciò che ha esistenza. In questo senso essa è quindi anteriore allo stesso Ricettacolo, allo stesso
} 
ricettacolo e chora, tuttavia rispetto allo schema poniamo al secondo posto il ricettacolo intelligibile, e la chora, implicata dall'ordinamento tramite corretto scuotimento, come precondizione spaziale di quello sensibile

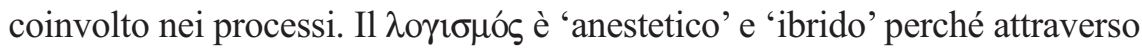
la privazione dai sensi $\mathrm{i}^{75}$ tenta di dare una forma a ciò che è amorfo, quindi applica categorie formali a ciò che, se in principio è 'senza ragione e senza misura', dopo l'intervento del dio resta comunque un divenire in massima parte disordinato e soggetto a perenne mutamento. E' su questa stabilizzazione geometrica che 'ragioniamo come in un sogno', ad esempio immaginando l'azione di triangoli elementari in ciò che ha moto perenne e caotico e che continuamente muta.

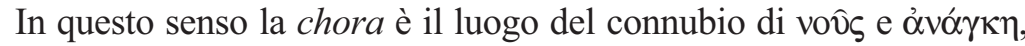
sede della generazione del cosmo ordinato in quanto spazio in cui tutto si colloca in un preciso ordine. Essa è ciò che noi possiamo concepire come il luogo in cui volta in volta si aggregano e disaggregano gli elementi resi il più possibile stabili dall'azione ordinatrice del dio ${ }^{76}$. E' da noi pensabile come spazio in cui la materia si informa, ma stiamo ragionando logisticamente, per approssimazione, nel tentativo di concepire la forma della causa errante, con un calcolo 'anestetico' e intermedio tra intelligenza e opinione su un qualcosa che è intermedio tra essere e generazione, in uno strano sogno da cui dobbiamo, ad un certo punto, risvegliarci.

\section{Dal disordine all'ordine: cosmogonia e materia nel Timeo}

Alzato di livello il ricettacolo nello schema delle aggiunte progressive, dobbiamo ora occuparci dei restanti passaggi. Ricordiamo innanzitutto quanto è detto da Timeo sugli 'elementi': quelli che chiamiamo

titolo di essere e generazione. Infatti, quando si muove, le materie prime occupano subito uno spazio proprio»; Migliori, La dialettica nel 'Timeo', p. 74.

${ }^{75}$ «In questa accezione, l'assenza di un riferimento sensoriale, che il testo le attribuisce, va intesa in senso forte, come segno di una realtà che né i sensi testimoniano, né il ragionamento garantisce adeguatamente»; Migliori, La dialettica nel 'Timeo', p. 74.

${ }^{76}$ In questo senso la chora, oltre ad essere la precondizione ontogonica della materia sensibile (in quanto principio di spazialità), sembra essere anche la precondizione gnoseologica della nostra riduzione geometrica della materia suddetta.

Educ. e Filos. Uberlândia, v. 23, n. 46, p. 263-308, jul./dez. 2009. 
'elementi' non solo non sono le 'lettere', ma neppure le 'sillabe' del cosmo ${ }^{77}$. Cosa s'intende allora distinguendo al punto 4) gli elementi e al 5) i corpi primi? Il testo ci dice che ci si serve di due 'strumenti', ossia i triangoli elementari. Il primo è l'isoscele e ha un'unica natura; il secondo è lo scaleno - o meglio 'il più bello'tra le infinite forme di scaleno - e ha natura molteplice. Questi sono propriamente gli elementi. Dalle combinazioni di tali triangoli elementari si generano i solidi geometrici che permettono la stabilizzazione dei protoelementi - o meglio 'protocorpi' 78 - nei quattro corpi primi: tre - fuoco aria e acqua - dallo scaleno, il quarto - la terra dall'isoscele ${ }^{79}$.

Modificato nei termini sopra esplicitati lo schema delle aggiunte progressive, possiamo ora tentare di sintetizzare il processo cosmogonico tracciando uno schema unitario comprensivo degli schemi verticale, orizzontale e delle aggiunte progressive da cui siamo partiti. Tale schema è sicuramente approssimativo ${ }^{80}$. Tuttavia, ci sembra che mostri in maniera efficace quanto è emerso dai 'terribili giochi' del Timeo presi in esame, ossia la stretta concatenazione delle trattazioni del processo di generazione del cosmo sensibile, la centralità di ordine e misura in tale processo, l'attività del principio ordinatore e la passività del principio materiale ${ }^{81}$, da principio (perlopiù) disordinato, poi persuaso e successivamente strutturato.

\footnotetext{
${ }^{77}$ Cfr. Timeo, 48 A 7-C 2; in greco si osserva il doppio senso di $\sigma \tau$ ๆ $\chi \varepsilon \hat{\imath} \alpha$, che significa sia 'lettere' sia 'elementi'.

${ }^{78}$ E' qui evidente il rischio di confusione nel parlare di proto-fuoco come 'protoelemento'. Se gli elementi sono i triangoli, i cosiddetti quattro elementi sono il realtà i corpi primi, e dunque nel loro stato predemiurgico dobbiamo concepirli come 'protocorpi'.

${ }^{79}$ La loro stabilità è poi bene illustrata in tutta la successiva 'sezione' sulle mescolanze e trasformazioni reciproche delle varie forme di fuoco, aria, acqua vista nell'indagine lessicale.

${ }^{80}$ Perché limitato alle prime due trattazioni di Timeo, con la sola menzione dell'imitazione illustrata nella terza parte e per la mancanza del proemio metafisico e del prologo drammaturgico, nonché della strana 'appendice' costituita dall'incompleto Crizia.

${ }^{81}$ La tradizione indiretta ci riferisce che Platone nelle sue "dottrine non scritte" chiamava questo principio con l'espressione "Diade indefinita di grande e piccolo", che esprime il principio del più e del meno, del troppo e del troppo poco, dell'eccesso e del difetto, del molteplice disordinato, e in vario modo include tutte le connotazioni del principio materiale che nel Timeo Platone ci ha presentato»; Reale, Timeo, Introduzione, p. 24.
} 


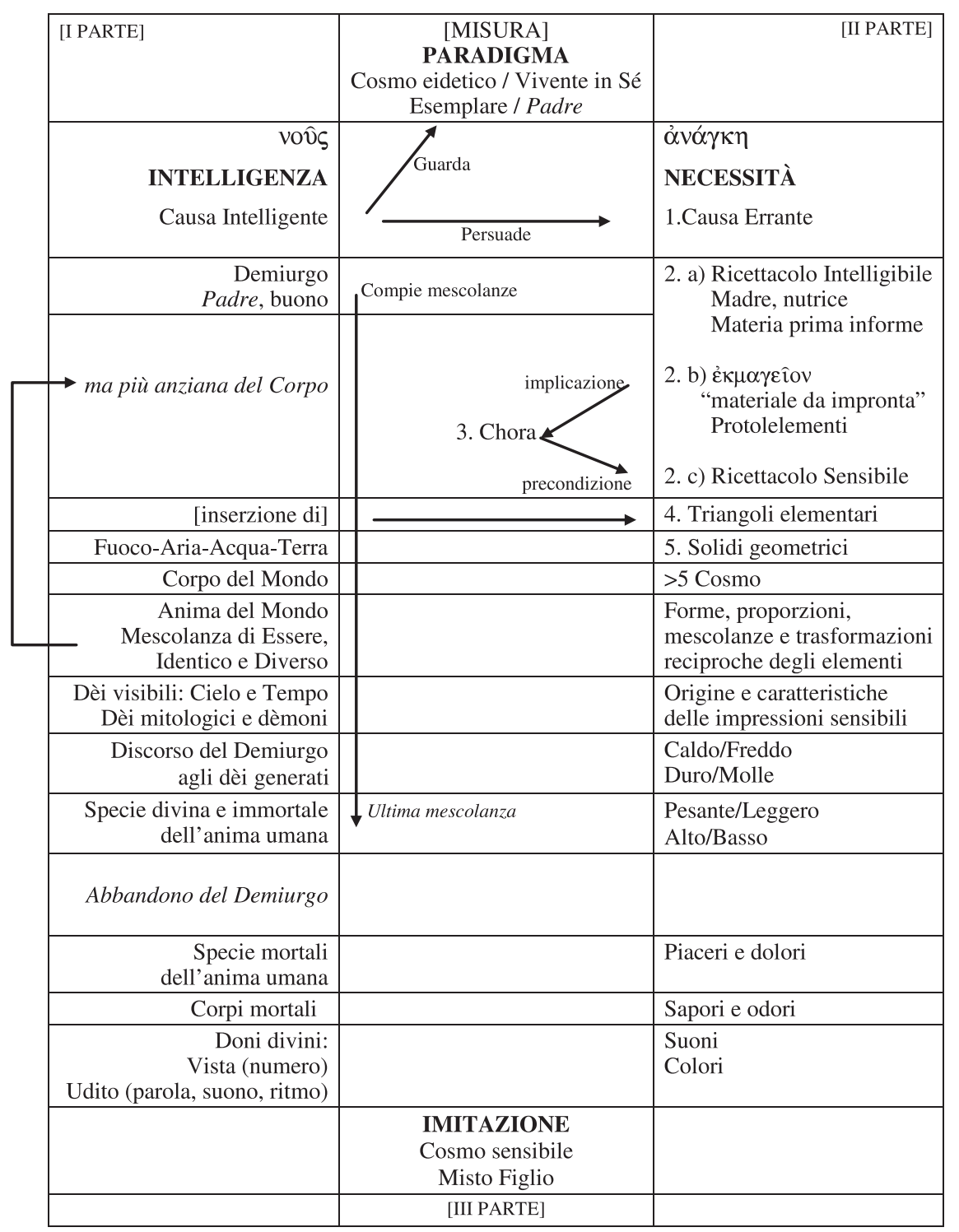


Alla luce dell'analisi condotta, possiamo dire che l'approccio 'ontologico' con cui ci siamo confrontati - seppure all'interno di un orizzonte ermeneutico comune - ci ha permesso di porre in evidenza la centralità del processo ordinatore nella generazione della realtà sensibile ${ }^{82}$. Sulla base di quanto è emerso dal rapporto tra Misura e misurato indagato nello screening lessicale, abbiamo visto come l'introduzione delle misure e delle proporzioni attraverso le mescolanze demiurgiche determini il graduale passaggio dal disordine all'ordine, in cui la materia funge da principio contrapposto e poi persuaso in momenti che sono 'successivi' in termini cosmogonici e non temporali (in quanto siamo ad un livello pre-temporale). Persino l'oscuro 'concetto-limite' ${ }^{83}$ di chora, letto come implicazione dell'ordinamento del ricettacolo intelligibile e precondizione spaziale o sostrato geometrico-strutturale del ricettacolo sensibile ${ }^{84}$, è risultato essere strettamente connesso, in quanto implicato ed implicante, a quello di ordine. La stessa dimensione dell'essere ci appare in qualche modo derivata da quella dell'ordine e della proporzione, letteralmente 'sub-ordinata' ad essa. Nella prima parte il demiurgo-ordinatore agisce sull'essere per mezzo di mescolanze, nella seconda il ricettacolo persuaso alla giusta misura nello scuotimento riceve forma e stabilità dai triangoli elementari, che agiscono in qualche maniera sui protoelementi (o meglio 'protocorpi') stabilizzandoli nei quattro corpi primi.

In altri termini, ai nostri occhi il processo generativo non è risultato soltanto e puramente ontogonico, ma ontogonico in quanto cosmogonico: l'essere stesso del cosmo è una conseguenza del passaggio dal disordine all'ordine.

\footnotetext{
${ }^{82}$ Il termine 'cosmogonia' significa letteralmente 'generazione di ordine'.

${ }^{83}$ In quanto è al «limite estremo della conoscibilità» (cfr. Migliori, La dialettica nel 'Timeo', p. 70) ed è il 'limite della materia' (cfr. Aristotele, Fisica, IV, 2, 209 a 36-209 b 5: «se il luogo è ciò che immediatamente contiene ciascun corpo, esso sarà, allora, un certo limite, sicché il luogo sembrerà essere la configurazione e la forma di ciascuna cosa, mediante cui sono limitati la grandezza e la materia della grandezza: giacché questo, appunto, è il limite di ciascuna cosa»).

${ }^{84}$ A proposito della distinzione dei livelli del ricettacolo, osserva Reale che «il principio materiale di cui parla il nostro dialogo non è la Diade nella sua globalità, ma solo quella sensibile. La Diade infatti ha un suo ruolo bene determinato anche nella sfera degli enti intelligibili e in quella degli enti intermedi come una sorta di "materia intelligibile"»; Reale, Timeo, Introduzione, pp. 24-5.
} 
La centralità di tale passaggio ci sembra pertanto una plausibile chiave di lettura del mito verosimile di Timeo e delle sue suggestive figure: l'intelligenza ordinatrice, la necessità errante e il cosmo ordinato che dal loro connubio si genera, ad imitazione di un Ordine Superiore.

E chi ammettesse questo principio della generazione del cosmo come principale, accettandolo da uomini saggi, l'ammetterebbe assai rettamente. Volendo infatti il dio che tutte le cose fossero buone, e che nulla fosse cattivo per quanto è possibile, prendendo quanto era visibile e non stava in quiete, ma si muoveva confusamente e disordinatamente, lo portò dal disordine all'ordine, giudicando questo del tutto migliore di quello. Infatti non è lecito a chi è ottimo di fare se non ciò che è bellissimo. ${ }^{85}$

\section{Bibliografia}

AA. VV., La struttura del dialogo platonico, a cura di G. Casertano, Napoli 2000. AA. VV., Gigantomachia, Convergenze e divergenze tra Platone $e$ Aristotele, ed. M. Migliori, Morcelliana, Brescia 2002.

AA. VV., La sapienza di Timeo. Riflessioni in margine al 'Timeo'di Platone, ed. L. M. Napolitano Valditara, Vita e Pensiero, Milano 2007.

AA. VV., Platone e Aristotele, Dialettica e logica, a cura di M. Migliori e A. Fermani, Morcelliana, Brescia 2008.

Brisson, L., Le même et l'autre dans la structure ontologique du Timee de Platon: un commentaire systematique du Timee de Platon, Paris 1974.

Cannarsa, M., La 'cornice letteraria' del 'Timeo', in La sapienza di Timeo, pp. 3-48. Cattanei, E., Aristotele e i "calcoli" dell'uomo saggio, in Dialettica e logica, pp. 61-86.

Derrida, J., Khôra, Galilée, Paris 1993, trad it. di F. Garritano, Chora, in Il segreto del nome, Jaca Book, Milano 1997.

Fronterotta, F., Platone, Timeo, Introduzione, traduzione e note di F. Fronterotta, B.U.R., Milano 2003

Kaplan, R., Zero. Storia di una cifra, BUR, Milano 1999, $2006^{4}$.

$\overline{{ }^{85} \text { Timeo, } 29 \text { E 4-30 A } 7 .}$

Educ. e Filos. Uberlândia, v. 23, n. 46, p. 263-308, jul./dez. 2009. 
Migliori, M., «Non entrichiè “ageometretos”». Il connubio tramatematiche e filosofia nell'insegnamento di Platone, in Filosofia, logica, matematica dal periodo classico al nostro secolo. Atti del convegno dell'I.R.R.S.A.E. Marche, Ancona 25-27 marzo 1993, a cura di A. Repola Boato, Quaderni di "Innovazione Scuola", 18 (1994), pp. 15-40, p. 22.

Migliori, M., Platone, Filebo, Introduzione, traduzione, note, apparati e appendice bibliografica di M. Migliori, Rusconi, Milano 1995.

Migliori, M., L'uomo fra piacere, intelligenza e Bene, introduzione di Th. A. Szlezák,Vita e Pensiero, Milano 1993.

Migliori, M., Tra polifonia e 'puzzle'. Esempi di rilettura del 'gioco'filosofico di Platone, in La struttura del dialogo platonico, pp. 171-212.

Migliori, M., Comment Platon ècrit-il? Example d'une écriture à caractère 'protreptique', in M. Fattal (sous la direction de), La philosophie de Platon, Paris 2005, pp. 83-118 (versione italiana: Come scrive Platone. Esempi di una scrittura a carattere 'protrettico', "Annali della Facoltà di Lettere e Filosofia dell'Università di Macerata», 37 (2004), pp. 249-77).

Migliori, M., Ontologia e materia. Un confronto tra il Timeo di Platone e il De generatione et corruptione di Aristotele, in Gigantomachia, pp. 35-104.

Migliori, M., La dialettica nel 'Timeo', in La sapienza di Timeo, pp. 49-107. Reale, G., Storia della filosofia antica, 5 Voll., Vita e Pensiero, Milano 1997. Reale, G., Platone, Timeo, Introduzione, traduzione, note, apparati e appendice iconografica di Giovanni Reale, Rusconi, Milano 1994, Bompiani 2000.

Ragazzoni, S., Nel nome di Chōra. Da Derrida a Platone e al di là, il melangolo, Genova 2008.

Sallis, J., Chorology: on beginning in Plato's Timaeus, Bloomington, Indianapolis, Indiana University Press, 1999.

Tarán, L., Academica: Plato, Philip of Opus, and the pseudo-Platonic Epinomis, American Philosophical Society, Philadelphia 1975. 